\title{
Impact of hemodialysis and post-dialysis period on granular activity levels
}

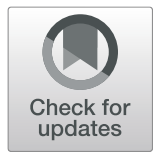

John W. Larkin ${ }^{1,2^{*}}$ D, Maggie Han ${ }^{1,3}$, Hao Han², Murilo H. Guedes ${ }^{1}$, Priscila Bezerra Gonçalves ${ }^{4}$, Carlos Eduardo Poli-de-Figueiredo ${ }^{5}$, Américo Lourenço Cuvello-Neto ${ }^{6}$, Ana Beatriz L. Barra7, Thyago Proença de Moraes ${ }^{1}$, Len A. Usvyat ${ }^{2}$, Peter Kotanko ${ }^{3,8}$, Maria Eugenia F. Canziani ${ }^{9}$, Jochen G. Raimann ${ }^{3}$, Roberto Pecoits-Filho ${ }^{1}$ and For the HDFIT Study Investigators

\begin{abstract}
Background: Physical activity (PA) is typically lower on hemodialysis (HD) days. Albeit intradialytic inactivity is expected, it is unknown whether recovery after HD contributes to low PA. We investigated the impact of HD and post-HD period on granular PA relative to HD timing.

Methods: We used baseline data from the HDFIT trial conducted from August 2016 to October 2017. Accelerometry measured PA over 1 week in patients who received thrice-weekly high-flux HD (vintage 3 to 24 months), were clinically stable, and had no ambulatory limitations. PA was assessed on HD days ( 0 to $\leq 24$ $\mathrm{h}$ after start HD), first non-HD days ( $>24$ to $\leq 48 \mathrm{~h}$ after start HD) and second non-HD day ( $>48$ to $\leq 72 \mathrm{~h}$ after start HD). PA was recorded in blocks/slices: $4 \mathrm{~h}$ during HD, 0 to $\leq 2 \mathrm{~h}$ post-HD (30 min slices), and $>2$ to $\leq 20 \mathrm{~h}$ post-HD ( $4.5 \mathrm{~h}$ slices). Blocks/slices of PA were captured at concurrent/parallel times on first/second non-HD days compared to HD days.
\end{abstract}

Results: Among 195 patients (mean age $53 \pm 15$ years, 71\% male), step counts per 24-h were $3919 \pm 2899$ on HD days, $5308 \pm 3131$ on first non-HD days $(p<0.001)$, and $4926 \pm 3413$ on second non-HD days $(p=0.032)$. During concurrent/parallel times to HD on first and second non-HD days, patients took 1308 and 1128 more steps (both $p$ < 0.001). Patients took 276 more steps and had highest rates of steps/hour 2-h post-HD versus same times on first non-HD days (all $p<0.05$ ). Consistent findings were observed on second non-HD days.

Conclusions: PA was higher within 2-h of HD versus same times on non-HD days. Lower PA on HD days was attributable to intradialytic inactivity. The established PA profiles are of importance to the design and development of exercise programs that aim to increase activity during and between HD treatments.

Trial registration: HDFIT was prospectively registered 20 April 2016 on ClinicalTrials.gov (NCT02787161)

Keywords: High-flux hemodialysis, Physical activity, Steps, Moderate-to-vigorous activity, Accelerometry, End stage kidney disease

\footnotetext{
* Correspondence: John.Larkin@fmc-na.com

'School of Medicine, Pontifícia Universidade Católica do Paraná, Imaculada

Conceição, 1155, Curitiba, PR 80215-901, Brazil

${ }^{2}$ Global Medical Office, Fresenius Medical Care, Waltham, MA, USA

Full list of author information is available at the end of the article
}

(c) The Author(s). 2020 Open Access This article is licensed under a Creative Commons Attribution 4.0 International License, which permits use, sharing, adaptation, distribution and reproduction in any medium or format, as long as you give appropriate credit to the original author(s) and the source, provide a link to the Creative Commons licence, and indicate if changes were made. The images or other third party material in this article are included in the article's Creative Commons licence, unless indicated otherwise in a credit line to the material. If material is not included in the article's Creative Commons licence and your intended use is not permitted by statutory regulation or exceeds the permitted use, you will need to obtain permission directly from the copyright holder. To view a copy of this licence, visit http://creativecommons.org/licenses/by/4.0/ The Creative Commons Public Domain Dedication waiver (http://creativecommons.org/publicdomain/zero/1.0/) applies to the data made available in this article, unless otherwise stated in a credit line to the data. 


\section{Background}

A physically active lifestyle can improve and maintain health in children, adults, and populations with disabilities or chronic diseases [1-4]. People with end stage kidney disease (ESKD) typically perform low levels of physical activity (PA) (e.g. < 5000 steps/day), placing them at higher risk for obesity, diminished quality of life, cardiovascular diseases, and mortality [3-8]. Inactivity in ESKD is likely influenced by high prevalence of physical disabilities and comorbidities, and social, environmental, and patient characteristics [3, 4, 9-15]. Albeit lifesaving, dialysis can be associated with symptoms such as nausea, body aches, and fatigue impacting PA [12, 16, 17]. Understanding profiles and drivers of PA in ESKD is important to find optimal treatment options and design interventions.

Profiles of objectively measured PA are loosely defined in ESKD. Studies in hemodialysis (HD) populations using pedometers/accelerometers report average PA levels range from 2446 to 8454 steps/day; PA is lower on $\mathrm{HD}$ days versus non-HD days $[6,7,18-25]$. and PA decreases with longer vintage $[18,24]$. A limitation of most studies conducted to date is they assessed PA by calendar days (i.e. 0:00-23:59 h) and have not assessed PA relative to the timing of $\mathrm{HD}[2,5-7,18-27]$. It is unknown if recovery after HD may influence PA on HD days in addition to requirements of sitting/lying during HD. With the potential impacts of HD on PA, granular assessments are needed to find timepoints for risk assessment and interventions.

PA was measured via accelerometry in HD patients during the baseline period of the HDFIT randomized controlled trial (RCT) $[28,29]$. Various types of PA were assessed in 24-h periods relative to the start of HD (0to- $\leq 24 \mathrm{~h}$ after start $\mathrm{HD}$ ) and concurrent/parallel periods to HD days on the subsequent first and second non-HD days (>24-to- $\leq 48$ and $>48$-to- $\leq 72 \mathrm{~h}$ after start HD, respectively). PA data was captured in granular slices. Our primary hypothesis was granular slices of PA would be lower on HD days, particularly immediately after HD, versus concurrent time segments on the first and second non-HD days.

\section{Methods}

\section{Trial design}

HDFIT was a prospective, multi-center, unblinded, RCT studying the impact of modality on objective PA [28]. The HDFIT protocol and trial design has been previously published [29]. After a run-in baseline period up to 4 weeks, eligible participants were randomized in a 1 : 1 ratio to high-volume online hemodiafiltration (HDF) or high-flux HD for a 6-month interventional period and 12-month observational follow-up. This ancillary study investigated PA profiles during the run-in baseline period (ClinicalTrials.gov Identifier: NCT02787161; registration date 20 April 2016).

\section{Setting and participants}

Fourteen dialysis centers in Brazil were activated for recruitment. The Center for Epidemiology and Clinical Research (EPICENTER) academic clinical research organization (ACRO) based at Pontifícia Universidade Católica do Paraná (PUCPR) managed the trial.

Patients provided written informed consent before study activities. Trial included adults (age $\geq 18$ years) who initiated renal replacement therapy between 3 and 24 months prior to randomization, were treated with thrice-weekly HD, used a fistula/graft or permanent catheter, had previous $\mathrm{Kt} / \mathrm{V} \geq 1.2$, and were considered clinically stable. Patients with severely limited mobility/ ambulation, poor treatment adherence, or a life expectancy of $<3$ months because of non-renal comorbidities were excluded.

\section{Ethical considerations}

The protocol, consent form, and study documents were approved PUCPR ethics review board (central application\# 54926916.7.1001.0020; approval\# 1.538.784). Trial was conducted in accordance with the Declaration of Helsinki.

\section{Procedures and interventions}

PA was continuously measured over 7 days using a validated waist worn tri-axial accelerometer ActiGraph $^{\text {tw }}$ wGT3X-BT model, Pensacola, USA) [19, 30-33]. Patients were instructed to remove the accelerometer during sleep and bathing, and record their HD, sleep, and bathing times during monitoring.

Patient demographics (age, sex, race), clinical, laboratory, and social parameters were recorded from medical records and/or assessments. Clinical characteristics applicable to this ancillary study included etiology of ESKD, comorbidities, access type, height, pre-HD/postHD/estimated dry weight, pre-HD/post-HD systolic and diastolic blood pressure (SBP/DBP), pulse, and HD parameters. Educational level, family income, employment status, transportation method to clinic, and distance from clinic to the patient's residence was also captured.

Recent monthly values for pre-/post-HD blood urea nitrogen (BUN) and hemoglobin, and quarterly values for albumin, potassium, calcium, phosphate, intact parathyroid hormone (iPTH) were captured from the medical records. HD adequacy (single-pool $\mathrm{Kt} / \mathrm{V}$ ) was calculated from BUN [34].

\section{Endpoints}

Primary endpoints were the difference in steps and moderate-to-vigorous PA (MVPA) per $24 \mathrm{~h}$ and between 
prespecified periods after dialysis on HD days versus concurrent/parallel periods at the same time on subsequent first or second non-HD days; similar assessments were performed for light PA, sedentary time, and metabolic rate (MET) levels. Secondary endpoints were the difference in steps and MVPA per $24 \mathrm{~h}$ and in prespecified periods after the start of dialysis: i) on the first versus second or third HD session of the week, and ii) HD start before versus after $1500 \mathrm{~h}$. A further exploratory analysis of the difference in sedentary time per $24 \mathrm{~h}$ and in prespecified periods was performed in patients starting HD before versus after $1500 \mathrm{~h}$.

An exploratory analysis was performed for a subgroup assessment of the difference in step counts and sedentary time in the prespecified periods $24 \mathrm{~h}$ after the start of HD stratified by transportation category for public (use of public transportation or walking) versus car (use of a family car, taxi, or ambulance) transportation. Also, sub-group analysis was performed to calculate the difference in step counts and sedentary time per $24 \mathrm{~h}$ after HD initiation by HD shifts starting before/after $1500 \mathrm{~h}$ stratified by age category $(<65$ versus $\geq 65$ years old) and family income category $(<2$ versus $>2$ minimum wages).

\section{Statistical methods}

\section{Physical activity analysis}

Raw accelerometer data were uncompressed using ActiLife v6.13.3 software (ActiGraph LLC, Pensacola, USA) and bandpass filtered using default filter with a $60 \mathrm{~s}$ epoch length. Data validation and filtering of non-wear time measurements was performed via vector magnitude and Choi 2011 algorithm [35]. We used a 60-min window for consecutive zeros/non-zeros [36].

PA data was cleaned, sliced, computed and exported using a custom coded file denoting slices based off start and end time of each HD session. Freedson VM3 Combination (2011) algorithm was used to calculate energy expenditure [37]. Raw acceleration cut points were: Sedentary $=0-99 \mathrm{cpm} \quad(\mathrm{CPM}) ; \quad$ Light $=100-2689 \quad \mathrm{CPM}$; Moderate $=2690-6166$ CPM; Vigorous $=6167-9642$ CPM; Very Vigorous = 9643-infinity CPM [37]. Freedson Adult 1998 algorithm was used to calculate MET levels [38]. MET levels represent the amount of energy expended per unit time in reference to sitting/resting MET level of 1 . If monitor was worn during sleep, custom export files were coded to exclude the data using patient-reported sleep times.

Extraction of 24-h periods of PA data was performed in 3 blocks consisting of 9 slices: Block A) HD/concurrent non-HD period with 1 slice of PA data, Block B) $\leq 2-\mathrm{h}$ post-HD/concurrent non-HD period with four data slices of $30 \mathrm{~min}$ each, and Block C) $>2$-to- $\leq 20$-h post$\mathrm{HD} /$ concurrent non-HD period with four data slices of $4.5 \mathrm{~h}$ each (Fig. 1).

\section{Analysis of endpoints}

Primary endpoints were analyzed by ANOVA and pooled two-way t-tests. Granular PA data for steps and minutes of MVPA was normalized to a rate per hour metric for visualization of data in prespecified periods. Secondary endpoints (i) and (ii) were compared using paired and unpaired t-tests, respectively.

Exploratory sub-group analysis of step counts and sedentary time in prespecified periods on HD days by

\section{Design for the Capture of Physical Activity Levels}

Activity tracking over 7 days starting at the initiation of hemodialysis (HD), or concurrent/parallel times on non-HD days and the long interdialytic day

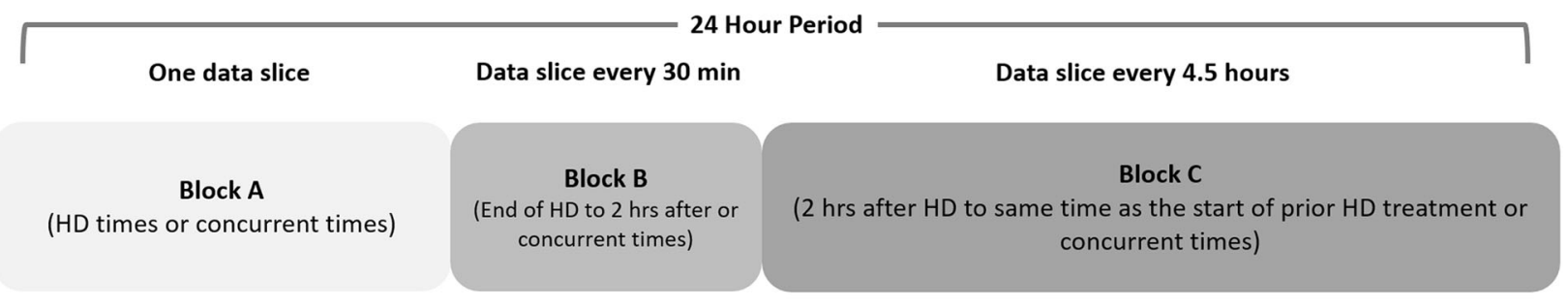

24-hour Period After Start of HD, or Concurrent Times on Non-HD Days, Used for Assessment of PA Profiles at Baseline

Fig. 1 Schematic of the design for the export and analysis of physical activity levels in in reference to the timing of the start of HD, or concurrent/ parallel times on non-HD days. Data was capture over $24 \mathrm{~h}$ periods in 3 blocks. The blocks were sliced to periods to investigate granular profiles after HD 
transportation type category were compared using unpaired t-tests. Comparisons for sub-group analysis of steps counts and sedentary time per $24 \mathrm{~h}$ on HD days in HD shifts starting before/after $1500 \mathrm{~h}$ by age and family income categories were performed with unpaired t-tests.

\section{Results}

\section{Patient characteristics}

HDFIT enrolled 195 patients from 13 of 14 sites throughout Brazil during August 2016 to October 2017 (Fig. 2). On average, patients were 53 years old, $71 \%$ male, $59 \%$ white race, $35 \%$ had diabetes, $17 \%$ had coronary artery disease, $8 \%$ had congestive heart failure; $89 \%$ used an arteriovenous fistula/graft and 55\% had ESKD attributable to diabetes/hypertension. Family income was 2 -to-10 times the Brazilian minimum wage in $73 \%$ and below that range in $18 \%$. Fifty-six percent completed high school or higher. Seventy-two percent resided $\geq 7$ $\mathrm{km}$ from the dialysis clinic; $43 \%$ drove by car and 33\% by public transportation to the HD clinic. Mean albumin was $4.0 \pm 0.4 \mathrm{~g} / \mathrm{dL}$, phosphate $5.3 \pm 1.4 \mathrm{mg} / \mathrm{dL}$, iPTH
$350.5 \pm 290.0 \mathrm{pg} / \mathrm{mL}$, hemoglobin $11.1 \pm 1.6 \mathrm{~g} / \mathrm{dL}$, and Kt/V $1.5 \pm 0.4$ (Table 1).

\section{Profiles of physical activity}

Among 195 HD patients with PA monitored, 176 had valid data. On average, patients took $4654 \pm 3468$ steps, their energy expenditure rate was $1.1 \pm 0.2 \mathrm{MET}$ (kcal/ $\mathrm{kg} / \mathrm{hr}$ ), and they performed $23.9 \pm 32.6 \mathrm{~min}$ of MVPA, $361.2 \pm 134.5 \mathrm{~min}$ of light PA, and $488.0 \pm 148.0 \mathrm{~min}$ of sedentary time per 24-h period (Table 2). ANOVA of PA on HD days and the first, and second non-HD days revealed differences between groups for steps $(p<0.001)$, MET $(p=0.043)$, MVPA $(p<0.001)$, light PA $(p<$ $0.0001)$ and sedentary time $(p<0.001)$ per $24 \mathrm{~h}$.

On the first non-HD days, patients performed 1389 more steps $(p=0.032), 7.2$ more minutes of MVPA $(p=$ $0.021), 107.9$ more minutes of light PA $(p<0.0001)$, and 69.8 fewer minutes of sedentary time $(p<0.0001)$ per 24-h compared to HD days. MET levels were the same on the first non-HD days versus HD days (Table 2).

On the second non-HD day, patients performed 1007 more steps $(p<0.001), 0.02$ lower MET $(p=0.027), 92.2$

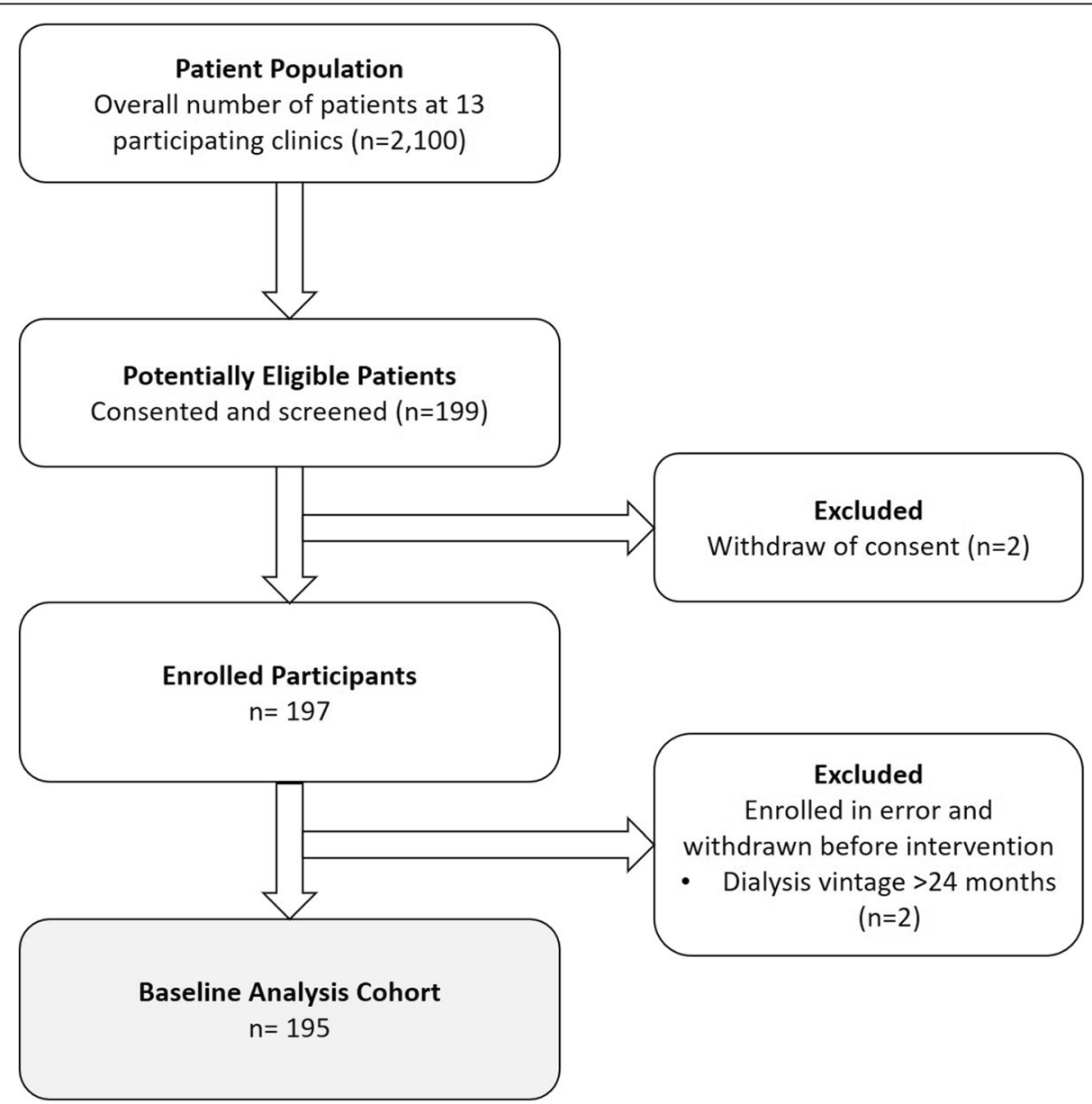

Fig. 2 Participant Flow Diagram 
Table 1 Patient Characteristics

\begin{tabular}{|c|c|c|c|}
\hline Parameter & Category & Total N & Mean $( \pm \mathrm{SD})$ or $\mathrm{N}$ and $\%$ \\
\hline Age (years) & & 195 & $52.98( \pm 15.08)$ \\
\hline Male (\%) & & 195 & $139(71)$ \\
\hline \multirow[t]{2}{*}{ Race } & White (\%) & 195 & $115(59)$ \\
\hline & Other (\%) & & $80(41)$ \\
\hline \multirow[t]{3}{*}{ Comorbidities } & Diabetes (\%) & 195 & $68(35)$ \\
\hline & Coronary artery disease (\%) & & $33(17)$ \\
\hline & Congestive heart failure (\%) & & $15(8)$ \\
\hline \multirow[t]{3}{*}{ Dialysis access type } & Arteriovenous fistula (\%) & 195 & $164(84)$ \\
\hline & Arteriovenous graft (\%) & & $9(5)$ \\
\hline & Permanent catheter (\%) & & $22(11)$ \\
\hline \multirow[t]{9}{*}{ Etiology of CKD/ESKD } & Diabetic nephropathy (\%) & 195 & $56(29)$ \\
\hline & Hypertensive nephrosclerosis (\%) & & $51(26)$ \\
\hline & Chronic glomerulonephritis (\%) & & $32(16)$ \\
\hline & Interstitial nephritis (\%) & & $7(4)$ \\
\hline & Chronic pyelonephritis (\%) & & $7(4)$ \\
\hline & Polycystic Kidney Disease (\%) & & $11(6)$ \\
\hline & Lupus Nephritis (\%) & & $5(3)$ \\
\hline & Other (\%) & & $11(6)$ \\
\hline & Unknown (\%) & & $15(8)$ \\
\hline \multirow[t]{4}{*}{ Family income level } & Above 10 minimum wages (\%) & 195 & $17(9)$ \\
\hline & 4 to 10 minimum wages (\%) & & $54(28)$ \\
\hline & 2 to 4 minimum wages (\%) & & $88(45)$ \\
\hline & Less than 2 minimum wages (\%) & & $36(18)$ \\
\hline \multirow[t]{4}{*}{ Education level } & Complete university (\%) & 195 & $39(20)$ \\
\hline & Complete high school (\%) & & $70(36)$ \\
\hline & Complete elementary (\%) & & $53(27)$ \\
\hline & Incomplete elementary (\%) & & $33(17)$ \\
\hline \multirow[t]{5}{*}{ Distance from home to clinic } & Greater than $25 \mathrm{Km}(\%)$ & 195 & $48(25)$ \\
\hline & Between 13 and $25 \mathrm{Km}(\%)$ & & $47(24)$ \\
\hline & Between 7 and 13 Km (\%) & & $46(24)$ \\
\hline & Less than $7 \mathrm{Km}(\%)$ & & $52(27)$ \\
\hline & Missing (\%) & & $2(1)$ \\
\hline \multirow[t]{5}{*}{ Transportation type to clinic } & Family car (\%) & 195 & $84(43)$ \\
\hline & Public transportation (\%) & & $65(33)$ \\
\hline & Ambulance (\%) & & $32(16)$ \\
\hline & Taxi (\%) & & $9(5)$ \\
\hline & Walking (\%) & & $5(3)$ \\
\hline \multicolumn{2}{|l|}{ Height (cm) } & 195 & $168.13( \pm 8.42)$ \\
\hline \multicolumn{2}{|l|}{ Estimated dry weight (Kg) } & 194 & $75.25( \pm 15.92)$ \\
\hline \multicolumn{2}{|l|}{ Pre-dialysis weight (kg) } & 194 & $77.78( \pm 15.98)$ \\
\hline \multicolumn{2}{|l|}{ Post-dialysis weight (kg) } & 193 & $75.51( \pm 15.79)$ \\
\hline \multicolumn{2}{|l|}{ Pre-dialysis SBP (mmHg) } & 193 & $153.19( \pm 23.7)$ \\
\hline \multicolumn{2}{|l|}{ Pre-dialysis DBP (mmHg) } & 193 & $80.93( \pm 13.43)$ \\
\hline \multicolumn{2}{|c|}{ Pre-dialysis pulse (beats per minute) } & 194 & $75.58( \pm 12.61)$ \\
\hline
\end{tabular}


Table 1 Patient Characteristics (Continued)

\begin{tabular}{|c|c|c|c|}
\hline Parameter & Category & Total N & Mean $( \pm S D)$ or $\mathrm{N}$ and $\%$ \\
\hline Post-dialysis SBP (mmHg) & & 192 & $148.14( \pm 23.01)$ \\
\hline Post-dialysis DBP (mmHg) & & 190 & $77.44( \pm 13.37)$ \\
\hline Post-dialysis pulse (beats per minute) & & 189 & $74.39( \pm 11.93)$ \\
\hline \multirow[t]{9}{*}{ Laboratories } & Pre-HD BUN (mg/dL) & 189 & $58.2(13.1)$ \\
\hline & Post-HD BUN (mg/dL) & 195 & $17.1(7.7)$ \\
\hline & $\mathrm{Kt} N$ & 194 & $1.5(0.4)$ \\
\hline & Albumin $(g / d L)$ & 192 & $4.0(0.4)$ \\
\hline & Potassium (mEq/L) & 195 & $5.2(0.8)$ \\
\hline & Calcium (mg/dL) & 175 & $9.0(0.7)$ \\
\hline & Phosphate (mg/dL) & 195 & $5.3(1.4)$ \\
\hline & Intact parathyroid hormone $(\mathrm{pg} / \mathrm{mL})$ & 190 & $350.5(290.0)$ \\
\hline & Hemoglobin $(g / d L)$ & 193 & $11.1(1.6)$ \\
\hline
\end{tabular}

Descriptive statistics are presented by parameter and category as applicable. The number of patients with valid data for each parameter are detailed in the third column (Total N); missing category data is detailed as applicable. The descriptive statistics in the fourth column are presented as the mean value \pm standard deviation (SD) for continuous variables or as the patient number (N) and percent (\%) for categorical variables

more minutes of light PA $(p<0.0001)$, and 35.8 fewer minutes of sedentary time $(p=0.026)$ per 24-h compared to HD days. Minutes of MVPA were similar for the second non-HD day versus HD days (Table 2).

\section{Granular profiles of physical activity}

Assessment of granular PA levels revealed, as expected, that sitting/lying during $\mathrm{HD}$ was associated with the most remarkable differences on HD days versus concurrent/parallel times on non-HD days. There were small increases in PA during the 2-h post-HD period versus the same times on non-HD days (Table 3 ).

At concurrent times to $\mathrm{HD}$ on the subsequent first non-HD day, patients performed an average of 1308 more steps $(p<0.001), 0.1$ higher MET $(p<0.001), 7.2$ more minutes of MVPA $(p<0.001), 88.1$ more minutes of light PA $(p<0.001)$, and 79.2 fewer minutes of sedentary time; on the second non-HD day patients performed 1128 more steps $(p<0.001), 0.1$ higher MET $(p<0.001)$, 5.5 more minutes of MVPA $(p<0.001), 82.4$ more minutes of light PA $(p<0.001)$, and 64.8 fewer minutes of sedentary time compared to HD days (Table 3). During
HD patients exhibited MET levels indicative of sitting still/resting $(\mathrm{MET}=1.0)$ and had $178.0 \mathrm{~min}$ of sedentary time, however they did perform low levels of some types of PA (97 steps, 0.3 min of MVPA, 13.3 min of light PA) that are likely attributable to slight movements during HD and could also be due to discrepancies in selfreported dialysis times captured from patient diaries.

Patients performed slightly more PA during the 2-h post-HD period on HD days versus concurrent times on subsequent non-HD days. During the 0-to-30, 31to-60, and 61-to-90-min post-HD slices on HD days, patients tended to take more steps, perform more MVPA/light PA, and spend less time in a sedentary state compared to concurrent times on both the first and second non-HD days. During the 91-to-120-min slice on HD days, patients tended to perform slightly more steps than concurrent times on the first nonHD days (Table 3 ). There was a small signal for patients performing lower PA during the $>2$-to- $\leq 6.5$ - $\mathrm{h}$ post-HD period on HD days versus the first non-HD days, where patients performed 13.9 fewer minutes of light PA $(p=0.009)$.

Table 2 Average Physical Activity (PA) Levels Per 24 Hours Overall and on HD Days, the First Non-HD Days and Second Non-HD Day

\begin{tabular}{|c|c|c|c|c|c|c|}
\hline \multirow[t]{2}{*}{ PA Measure $(N=176)$} & \multirow{2}{*}{$\begin{array}{l}\text { Overall } \\
( \pm S D) \\
\text { Per } 24 \text { Hrs }\end{array}$} & \multirow{2}{*}{$\begin{array}{l}\text { HD Day }( \pm S D) \\
\text { 0-to- } \leq 24 \text { Hrs } \\
\text { after Start HD }\end{array}$} & 1st Non-HD Day $( \pm S D)$ & \multirow[t]{2}{*}{$\begin{array}{l}\boldsymbol{P} \text {-Value } \\
\text { vs HD Day }\end{array}$} & \multirow{2}{*}{$\begin{array}{l}\text { 2nd Non-HD Day }( \pm S D) \\
>48 \text {-to- } \leq 72 \text { Hrs } \\
\text { after Start HD }\end{array}$} & \multirow{2}{*}{$\begin{array}{l}\boldsymbol{P} \text {-Value } \\
\text { vs HD } \\
\text { Day }\end{array}$} \\
\hline & & & $\begin{array}{l}>24 \text {-to- } \leq 48 \mathrm{Hrs} \\
\text { after Start HD }\end{array}$ & & & \\
\hline Steps Count & 4654 (3468) & 3919 (2899) & $5308(3131)$ & $<0.001$ & $4926(3413)$ & 0.032 \\
\hline MET (kcal/kg/Hr) & $1.08(0.19)$ & $1.09(0.17)$ & $1.09(0.23)$ & 0.797 & $1.07(0.18)$ & 0.027 \\
\hline MVPA Minutes & $23.9(32.6)$ & $20.3(27.0)$ & $27.5(31.3)$ & 0.021 & $24.3(28.3)$ & 0.175 \\
\hline Light PA Minutes & $361.2(134.5)$ & 294.5 (95.5) & $402.5(127.3)$ & $<0.0001$ & $386.7(149.5)$ & $<0.0001$ \\
\hline Sedentary Time Minutes & $488.0(148.0)$ & $523.2(118.2)$ & 453.4 (136.3) & $<0.0001$ & $487.3(176.2)$ & 0.026 \\
\hline
\end{tabular}


Table 3 Average Physical Activity (PA) Levels Per Predefined Granular Slice Period on HD Days, the First Non-HD Days and Second Non-HD Day

\begin{tabular}{|c|c|c|c|}
\hline \multicolumn{4}{|c|}{ Step Counts Per Predefined Granular Slice Period $(\boldsymbol{N}=176)$} \\
\hline \multirow{2}{*}{$\begin{array}{l}\text { Slice Period during/after HD or Concurrent } \\
\text { Time }\end{array}$} & HD Day $( \pm S D)$ & 1st Non-HD Day ( \pm SD) & 2nd Non-HD D \\
\hline & $\begin{array}{l}0 \text {-to- } \leq 24 \text { Hrs after Start } \\
H D\end{array}$ & $\begin{array}{l}>24-t o-\leq 48 \text { Hrs after Start } \\
H D\end{array}$ & $\begin{array}{l}>48-t o-\leq 72 H \\
H D\end{array}$ \\
\hline HD Period & $97(225)$ & $1405^{* * *}(1067)$ & $1225^{* * *}(1468)$ \\
\hline $0.0-\leq 0.5 \mathrm{Hr}$ Post-HD & $261(267)$ & $180^{* *}(179)$ & $137^{* * *}(177)$ \\
\hline$>0.5-\leq 1.0 \mathrm{Hr}$ Post-HD & $282(224)$ & $203^{* *}(299)$ & $147^{* * *}(211)$ \\
\hline$>1.0-\leq 1.5 \mathrm{Hr}$ Post-HD & $243(200)$ & $164^{* * *}(183)$ & $173^{*}(323)$ \\
\hline$>1.5-\leq 2.0 \mathrm{Hr}$ Post-HD & $179(181)$ & $142^{*}(149)$ & $148(218)$ \\
\hline$>2.0-\leq 6.5 \mathrm{Hr}$ Post-HD & $788(772)$ & $941(925)$ & $844(926)$ \\
\hline$>6.5-\leq 11.0 \mathrm{Hr}$ Post-HD & $608(729)$ & $718(926)$ & $704(1058)$ \\
\hline$>11.0-\leq 15.5 \mathrm{Hr}$ Post-HD & $964(1668)$ & $874(1343)$ & $1014(1749)$ \\
\hline$>15.5-\leq 20.0 \mathrm{Hr}$ Post-HD & 1257 (1352) & $1327(950)$ & 1384 (1082) \\
\hline
\end{tabular}

MET Rate Per Predefined Granular Slice Period $(N=176)$

Slice Period during/after HD or Concurrent Time

$$
\begin{aligned}
& \text { HD Period } \\
& 0.0-\leq 0.5 \mathrm{Hr} \text { Post-HD } \\
& >0.5-\leq 1.0 \mathrm{Hr} \text { Post-HD } \\
& >1.0-\leq 1.5 \mathrm{Hr} \text { Post-HD } \\
& >1.5-\leq 2.0 \mathrm{Hr} \text { Post-HD } \\
& >2.0-\leq 6.5 \mathrm{Hr} \text { Post-HD } \\
& >6.5-\leq 11.0 \mathrm{Hr} \text { Post-HD } \\
& >11.0-\leq 15.5 \mathrm{Hr} \text { Post-HD } \\
& >15.5-\leq 20.0 \mathrm{Hr} \text { Post-HD }
\end{aligned}
$$

HD Day ( \pm SD)

0 -to- $\leq 24$ Hrs after Start $H D$

$$
\begin{aligned}
& 1.0(0.03) \\
& 1.1(0.3) \\
& 1.1(0.2) \\
& 1.1(0.2) \\
& 1.1(0.2) \\
& 1.1(0.1) \\
& 1.1(0.2) \\
& 1.1(0.2) \\
& 1.1(0.1)
\end{aligned}
$$

Minutes of MVPA Per Predefined Granular Slice Period $(N=176)$

Slice Period during/after HD or Concurrent
Time

HD Day $( \pm S D)$

0-to- $\leq 24$ Hrs after Start $H D$

$$
\begin{aligned}
& \text { HD Period } \\
& 0.0-\leq 0.5 \mathrm{Hr} \text { Post-HD } \\
& >0.5-\leq 1.0 \mathrm{Hr} \text { Post-HD } \\
& >1.0-\leq 1.5 \mathrm{Hr} \text { Post-HD } \\
& >1.5-\leq 2.0 \mathrm{Hr} \text { Post-HD } \\
& >2.0-\leq 6.5 \mathrm{Hr} \text { Post-HD } \\
& >6.5-\leq 11.0 \mathrm{Hr} \text { Post-HD } \\
& >11.0-\leq 15.5 \mathrm{Hr} \text { Post-HD } \\
& >15.5-\leq 20.0 \mathrm{Hr} \text { Post-HD }
\end{aligned}
$$

\section{$0.3(10.8)$}

$1.3(1.8)$

$1.2(2.6)$

$0.9(1.7)$

$0.7(1.5)$

$3.6(7.4)$

$3.0(7.0)$

$6.6(13.7)$

$7.1(8.3)$

Minutes of Light PA Per Predefined Granular Slice Period $(N=176)$

Slice Period during/after HD or Concurrent Time

HD Day $( \pm S D)$

0-to- $\leq 24$ Hrs after Start $H D$

$$
\begin{aligned}
& \text { HD Period } \\
& 0.0-\leq 0.5 \mathrm{Hr} \text { Post-HD } \\
& >0.5-\leq 1.0 \mathrm{Hr} \text { Post-HD }
\end{aligned}
$$

$13.3(18.0)$
$13.6(5.5)$
$17.3(6.1)$

1st Non-HD Day ( \pm SD)

$>$ 24-to- $\leq 48$ Hrs after Start $H D$

$\begin{array}{ll}1.1^{* * *}(0.2) & 1.1^{* * *}(0.2) \\ 1.1(0.3) & 1.0^{* * *}(0.1) \\ 1.1(0.4) & 1.0^{* * *}(0.2) \\ 1.1(0.2) & 1.1(0.3) \\ 1.1(0.2) & 1.1(0.2) \\ 1.1(0.3) & 1.0(0.1) \\ 1.1(0.2) & 1.1(0.2) \\ 1.1(0.2) & 1.1(0.3) \\ 1.1(0.1) & 1.1(0.1)\end{array}$

1st Non-HD Day ( \pm SD)

$>$ 24-to- $\leq 48$ Hrs after Start $H D$

$7.5^{* * *}(1.6)$
$0.9(2.4)$
$1.1(2.0)$
$0.8(1.6)$
$0.7(1.4)$
$4.3(6.1)$
$3.7(4.5)$
$5.6(15.4)$
$6.4(12.0)$

1st Non-HD Day ( \pm SD)

$>$ 24-to- $\leq 48$ Hrs after Start $H D$

$101.4^{* * *}(41.6)$
$13.9(7.3)$
$13.5^{* * *}(7.1)$

2nd Non-HD Day ( \pm SD)

$>48$-to- $\leq 72$ Hrs after Start $H D$

$1.1^{* * *}(0.2)$

$1.0 * * *(0.1)$

$.0^{* * *}(0.2)$

$1(0.3)$

$(0.2)$

$1.1(0.2)$

$.1(0.1)$

2nd Non-HD Day ( \pm SD)

$>48$-to- $\leq 72$ Hrs after Start $H D$

$5.8^{* * *}(12.4)$

$0.6^{* *}(1.7)$

$0.5^{* *}(2.1)$

$0.8(2.9)$

$0.6(1.7)$

$4.3(9.2)$

$3.4(7.0)$

$6.1(13.7)$

$6.6(8.8)$

2nd Non-HD Day ( \pm SD)

$>48$-to- $\leq 72$ Hrs after Start $H D$

95.7*** $(50.6)$

13.1 (8.6)

$13.5^{* * *}(8.7)$ 
Table 3 Average Physical Activity (PA) Levels Per Predefined Granular Slice Period on HD Days, the First Non-HD Days and Second Non-HD Day (Continued)

\begin{tabular}{|c|c|c|c|}
\hline \multicolumn{4}{|c|}{ Step Counts Per Predefined Granular Slice Period $(\boldsymbol{N}=176)$} \\
\hline$>1.0-\leq 1.5 \mathrm{Hr}$ Post-HD & $16.1(6.9)$ & $12.9^{* * *}(6.6)$ & $12.9^{* * *}(9.2)$ \\
\hline$>1.5-\leq 2.0 \mathrm{Hr}$ Post-HD & $13.4(6.5)$ & $12.5(6.9)$ & $13.0(9.9)$ \\
\hline$>2.0-\leq 6.5 \mathrm{Hr}$ Post-HD & $72.5(43.6)$ & $86.4^{* *}(53.7)$ & $82.5(54.3)$ \\
\hline$>6.5-\leq 11.0 \mathrm{Hr}$ Post-HD & $56.7(45.2)$ & $60.8(47.8)$ & $61.9(49.2)$ \\
\hline$>11.0-\leq 15.5 \mathrm{Hr}$ Post-HD & $56.4(50.7)$ & $54.4(48.0)$ & $63.1(57.2)$ \\
\hline$>15.5-\leq 20.0 \mathrm{Hr}$ Post-HD & $89.6(56.9)$ & $92.1(42.6)$ & $94.7(53.3)$ \\
\hline \multicolumn{4}{|c|}{ Minutes of Sedentary Time Per Predefined Granular Slice Period $(N=176)$} \\
\hline \multirow{2}{*}{$\begin{array}{l}\text { Slice Period during/after HD or Concurrent } \\
\text { Time }\end{array}$} & HD Day ( \pm SD) & 1st Non-HD Day ( \pm SD) & 2nd Non-HD Day ( \pm SD) \\
\hline & $\begin{array}{l}0 \text {-to- } \leq 24 \text { Hrs after Start } \\
H D\end{array}$ & $\begin{array}{l}>24-t o-\leq 48 \text { Hrs after Start } \\
H D\end{array}$ & $\begin{array}{l}>48 \text {-to- } \leq 72 \text { Hrs after Start } \\
H D\end{array}$ \\
\hline HD Period & $178.0(57.2)$ & $98.8^{* * *}(49.6)$ & $113.2^{* * *}(61.4)$ \\
\hline $0.0-\leq 0.5 \mathrm{Hr}$ Post-HD & $14.6(6.0)$ & $14.6(7.7)$ & $15.9(9.1)$ \\
\hline$>0.5-\leq 1.0 \mathrm{Hr}$ Post-HD & $11.3(6.0)$ & $14.8^{* * *}(7.7)$ & $15.6^{* * *}(9.0)$ \\
\hline$>1.0-\leq 1.5 \mathrm{Hr}$ Post-HD & $12.7(7.3)$ & $15.6^{* * *}(7.1)$ & $16.0^{* * *}(9.7)$ \\
\hline$>1.5-\leq 2.0 \mathrm{Hr}$ Post-HD & $14.8(6.7)$ & $15.5(7.2)$ & $15.7(10.2)$ \\
\hline$>2.0-\leq 6.5 \mathrm{Hr}$ Post-HD & $125.3(59.4)$ & $118.3(56.5)$ & $128.9(64.1)$ \\
\hline$>6.5-\leq 11.0 \mathrm{Hr}$ Post-HD & $92.6(66.1)$ & $91.2(59.4)$ & $101.9(76.4)$ \\
\hline$>11.0-\leq 15.5$ Hr Post-HD & $64.9(47.4)$ & $61.9(45.2)$ & $75.6(65.7)$ \\
\hline$>15.5-\leq 20.0 \mathrm{Hr}$ Post-HD & $82.6(52.7)$ & $80.9(43.5)$ & $90.3(63.9)$ \\
\hline
\end{tabular}

***, $p<0.001 ; * *, p<0.01 ;{ }^{*}, p<0.05$

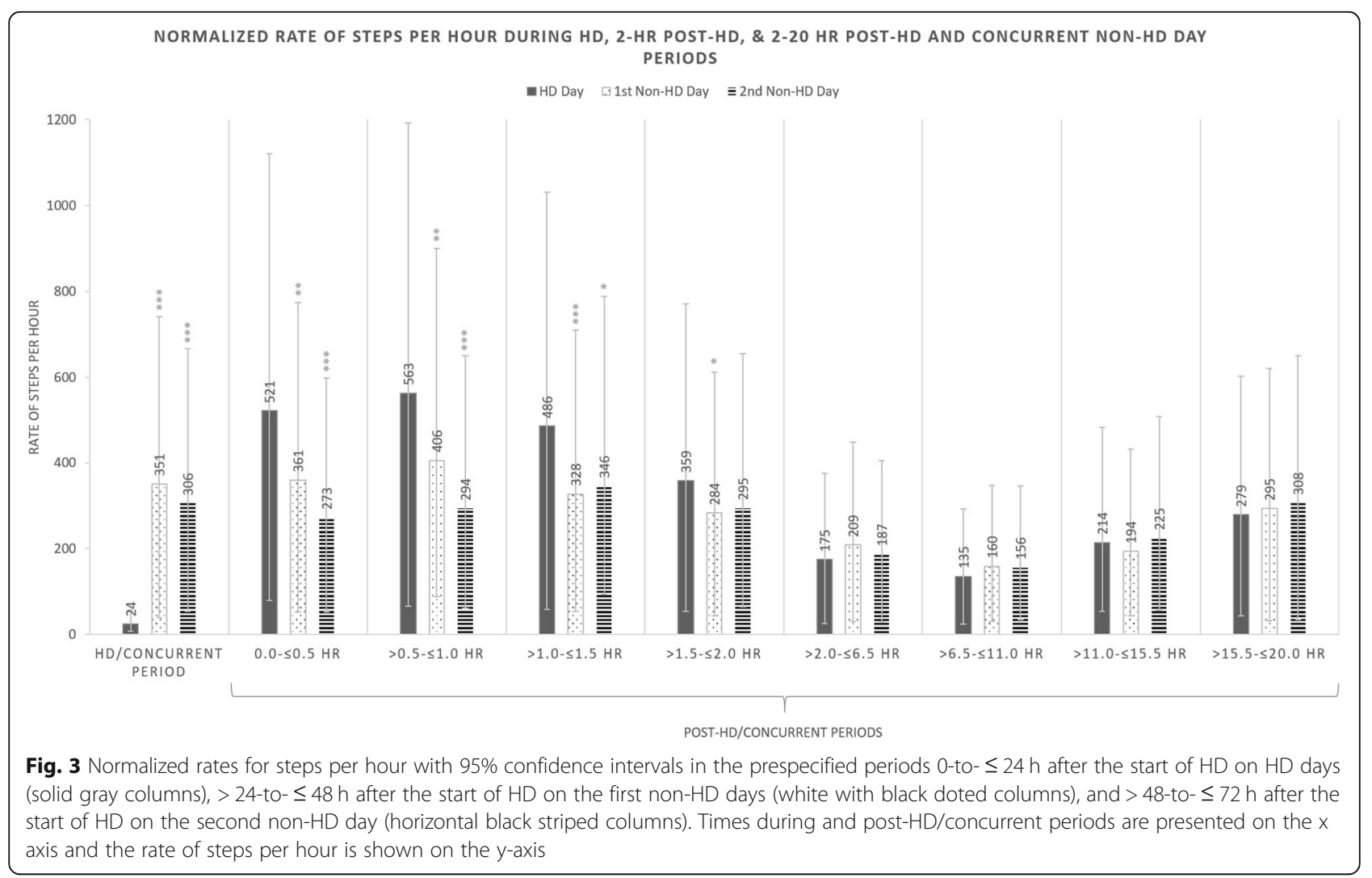




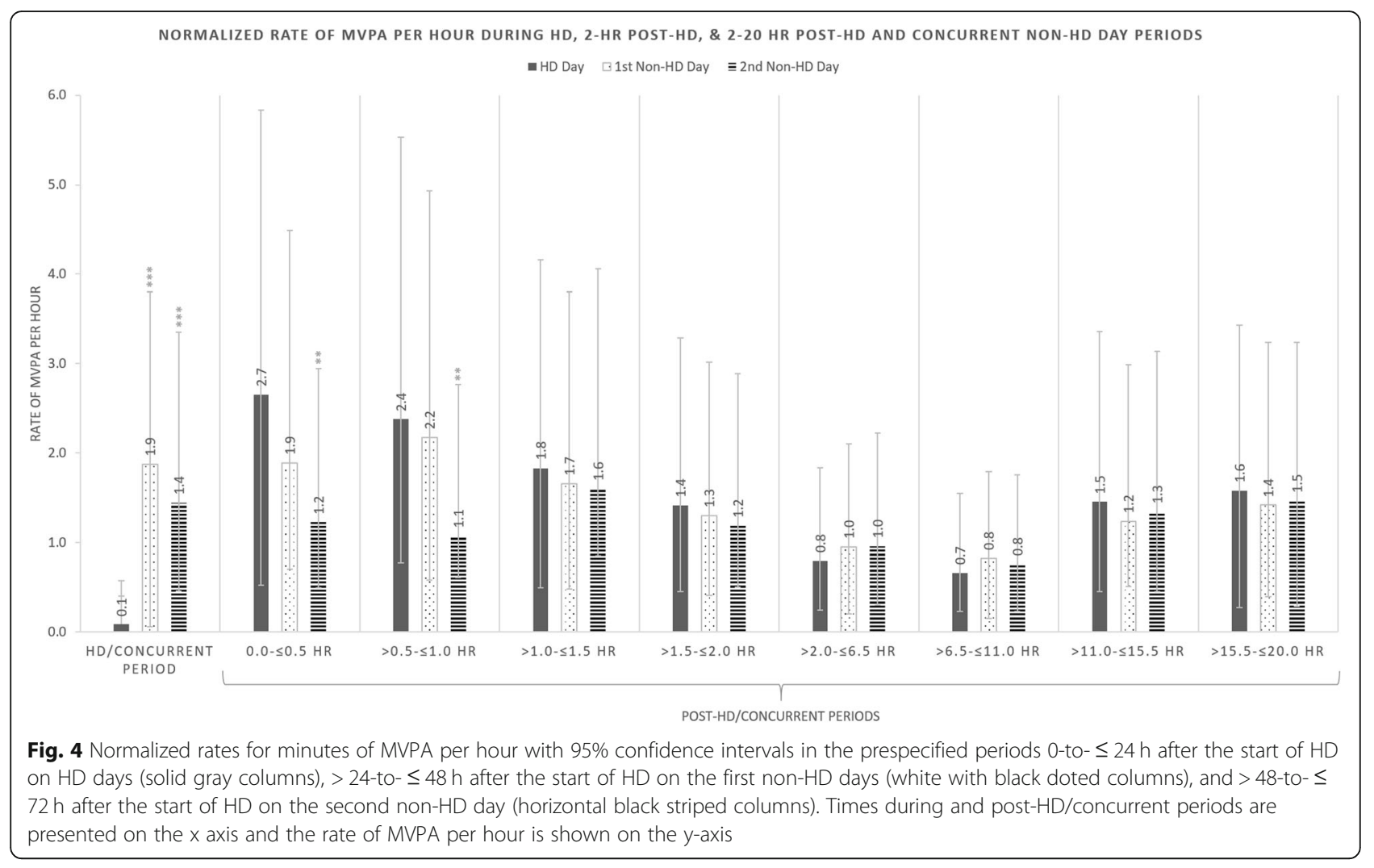

\section{Rates of granular physical activity}

Normalized rates of steps and minutes of MVPA performed per hour were calculated to visualize relative distinctions in prespecified periods (Figs. 3 and 4). Compared to the treatment time on HD days, patients performed rates of 327 and 282 more steps/hour and 1.8 and 1.4 more minutes of MVPA/hour during the concurrent times on the subsequent first and second nonHD days respectively (all $p<0.001$ ).

Patients generally performed higher rates of steps and minutes of MVPA in the 2-h post-HD period on HD days (Figs. 3 and 4). In the 0 -to-30-min post-HD period on HD days, patients performed rates of 161 and 248 more steps/hour compared to the first and second nonHD days respectively (both $p<0.01$ ); patients performed 1.4 more minutes of MVPA/hour on HD days versus the second-non-HD day $(p<0.01)$. During the 31-to-60, 61to-90, and 91-to-120-min slices of PA following HD, patients performed significantly more steps than concurrent times on both the first and second non-HD day (Fig. 3).

\section{Granular profiles of physical activity by transportation type}

An exploratory sub-group assessment of granular PA levels for step counts by categories of transportation type on HD days found patients using public transportation (category including public transportation or walking) performed on average a sum of 604 more steps during the $2-\mathrm{h}$ post-HD period versus patients using car transportation (category including family car, taxi, or ambulance) (Table 4). Patients using public transportation had on average 3.1 fewer minutes of sedentary time during only the predefined period $>1.5$-to$\leq 2 \mathrm{~h}$ after HD compared to patients using car transportation. No other differences were observed by transportation type for step counts and sedentary time in other prespecified timepoints in the $24 \mathrm{~h}$ during and after HD.

\section{Profiles of physical activity by Dialysis shift and day of the week}

Patients with HD sessions starting before $1500 \mathrm{~h}$ (first and second HD shift) performed 2157 fewer steps $(p<$ 0.0001), 18.6 fewer minutes of MVPA $(p<0.0001)$, and had 65.8 more minutes of sedentary time $(p<0.0001)$ per 24-h on HD days versus patients with HD sessions starting after $1500 \mathrm{~h}$ (third HD shift) (Table 5).

An exploratory sub-group analysis of step counts on HD days by shift and age category showed patients starting HD before $1500 \mathrm{~h}$ who were $<65$ years old performed 1668 more steps per $24 \mathrm{~h}$ after HD compared to patients $\geq 65$ years old $(p<0.0001)$, yet there were no significant distinctions in sedentary time (Table 6). There were no significant differences between step counts and 
Table 4 Average Physical Activity (PA) Levels Per Predefined Granular Slice Period on HD Days by Transportation Type

\begin{tabular}{|c|c|c|c|}
\hline \multicolumn{4}{|c|}{ Step Counts Per Predefined Granular Slice Period } \\
\hline $\begin{array}{l}\text { Slice Period during/ } \\
\text { after HD }\end{array}$ & $\begin{array}{l}\text { Steps on HD Days }( \pm S D) \text { with Public Transportation } \\
(N=66)\end{array}$ & $\begin{array}{l}\text { Steps on HD Days }( \pm S D) \text { with Car Transportation } \\
(N=110)\end{array}$ & $\begin{array}{l}\text { P-Value vs } \\
\text { Car }\end{array}$ \\
\hline HD Period & $128(323)$ & $78(136)$ & 0.156 \\
\hline $0.0-\leq 0.5 \mathrm{Hr}$ Post-HD & $364(355)$ & $201(174)$ & $<0.0001$ \\
\hline $\begin{array}{l}>0.5-\leq 1.0 \mathrm{Hr} \text { Post- } \\
\mathrm{HD}\end{array}$ & $400(269)$ & $210(154)$ & $<0.0001$ \\
\hline $\begin{array}{l}>1.0-\leq 1.5 \text { Hr Post- } \\
\text { HD }\end{array}$ & $320(234)$ & $197(161)$ & $<0.0001$ \\
\hline $\begin{array}{l}>1.5-\leq 2.0 \mathrm{Hr} \text { Post- } \\
\mathrm{HD}\end{array}$ & $259(218)$ & $132(135)$ & $<0.0001$ \\
\hline $\begin{array}{l}>2.0-\leq 6.5 \mathrm{Hr} \text { Post- } \\
\mathrm{HD}\end{array}$ & $880(660)$ & $732(830)$ & 0.227 \\
\hline $\begin{array}{l}>6.5-\leq 11.0 \text { Hr Post- } \\
\text { HD }\end{array}$ & $639(714)$ & $589(740)$ & 0.679 \\
\hline $\begin{array}{l}>11.0-\leq 15.5 \text { Hr Post- } \\
\text { HD }\end{array}$ & $1227(2006)$ & $808(1419)$ & 0.155 \\
\hline $\begin{array}{l}>15.5-\leq 20.0 \mathrm{Hr} \text { Post- } \\
\mathrm{HD}\end{array}$ & $1502(1524)$ & $1113(1227)$ & 0.094 \\
\hline \multicolumn{4}{|c|}{ Minutes of Sedentary Time Per Predefined Granular Slice Period } \\
\hline $\begin{array}{l}\text { Slice Period during/ } \\
\text { after HD }\end{array}$ & $\begin{array}{l}\text { Sedentary Minutes on HD Days }( \pm S D) \text { with Public } \\
\text { Transportation }(N=66)\end{array}$ & $\begin{array}{l}\text { Sedentary Minutes on HD Days }( \pm S D) \text { with Car } \\
\text { Transportation }(N=110)\end{array}$ & $\begin{array}{l}\text { P-Value vs } \\
\text { Car }\end{array}$ \\
\hline HD Period & $186.1(53.2)$ & $173.1(59.2)$ & 0.147 \\
\hline $0.0-\leq 0.5 \mathrm{Hr}$ Post-HD & $13.6(5.1)$ & $15.1(6.4)$ & 0.101 \\
\hline $\begin{array}{l}>0.5-\leq 1.0 \mathrm{Hr} \text { Post- } \\
\mathrm{HD}\end{array}$ & $11.7(5.7)$ & $11.0(6.2)$ & 0.473 \\
\hline $\begin{array}{l}>1.0-\leq 1.5 \mathrm{Hr} \text { Post- } \\
\mathrm{HD}\end{array}$ & $11.8(6.6)$ & $13.3(7.7)$ & 0.185 \\
\hline $\begin{array}{l}>1.5-\leq 2.0 \mathrm{Hr} \text { Post- } \\
\mathrm{HD}\end{array}$ & $12.8(6.2)$ & $15.9(6.8)$ & 0.003 \\
\hline $\begin{array}{l}>2.0-\leq 6.5 \text { Hr Post- } \\
\mathrm{HD}\end{array}$ & $119.3(54.5)$ & $128.9(62.2)$ & 0.307 \\
\hline $\begin{array}{l}>6.5-\leq 11.0 \text { Hr Post- } \\
\text { HD }\end{array}$ & $86.6(63.5)$ & $96.3(67.7)$ & 0.379 \\
\hline $\begin{array}{l}>11.0-\leq 15.5 \text { Hr Post- } \\
\text { HD }\end{array}$ & $68.8(44.4)$ & $62.6(49.1)$ & 0.457 \\
\hline $\begin{array}{l}>15.5-\leq 20.0 \text { Hr Post- } \\
\text { HD }\end{array}$ & $76.5(50.2)$ & $86.2(54.0)$ & 0.284 \\
\hline
\end{tabular}
transportation and walking

Table 5 Average Physical Activity (PA) Levels on HD Days Per 24 Hours by Dialysis Shift

\begin{tabular}{llll}
\hline PA Measure Per 24 Hrs on HD Days & HD start before 1500 Hrs $(\boldsymbol{N}=132)$ & HD start after 1500 Hrs $(\boldsymbol{N}=48)$ & $P$-Value vs HD before 1500 Hrs \\
\hline Step Counts $( \pm$ SD) & $3390(2313)$ & $5547(4613)$ & $<0.0001$ \\
Minutes of MVPA $( \pm$ SD) & $15.6(20.0)$ & $34.2(46.2)$ & $<0.0001$ \\
Minutes of Sedentary Time $( \pm$ SD) & $538.5(147.5)$ & $472.7(147.0)$ & $<0.0001$
\end{tabular}

PA levels on HD days by shift calculated based on each HD start time before or after $1500 \mathrm{~h}$. Among the 176 patients with accelerometry data, 4 patients received an HD treatment in both shifts during the 7 days of accelerometry 
Table 6 Average Physical Activity (PA) Levels on HD Days Per 24 Hours by Dialysis Shift, Age, and Family Income

\begin{tabular}{|c|c|c|c|c|}
\hline HD Shift & $\begin{array}{l}\text { PA Measure Per } 24 \text { Hrs } \\
\text { on HD Days }\end{array}$ & $\begin{array}{l}\text { Age }<65 \text { Years }(\boldsymbol{N}=130) \\
\text { Mean }( \pm \text { SD) N }\end{array}$ & $\begin{array}{l}\text { Age } \geq 65 \text { Years }(\boldsymbol{N}=46) \\
\text { Mean }( \pm S D) N\end{array}$ & $\begin{array}{l}\boldsymbol{P} \text { - } \\
\text { Value }\end{array}$ \\
\hline \multirow[t]{2}{*}{$\begin{array}{l}\text { HD start before } 1500 \mathrm{Hrs} \\
(N=132)\end{array}$} & Steps & 3897 (2356) 92 & 2229 (1727) 40 & $\begin{array}{l}< \\
0.0001\end{array}$ \\
\hline & Sedentary Time & 530.9 (148.7) 92 & $555.9(144.0) 40$ & 0.127 \\
\hline \multirow{2}{*}{$\begin{array}{l}\text { HD start after } 1500 \mathrm{Hrs} \\
(N=48)\end{array}$} & Steps & $5732(4134) 42$ & 4413 (6892) 7 & 0.237 \\
\hline & Sedentary Time & $463.0(140.9) 42$ & $531.7(172.2) 7$ & 0.053 \\
\hline HD Shift & $\begin{array}{l}\text { PA Measure Per } 24 \text { Hrs on } \\
\text { HD Days }\end{array}$ & $\begin{array}{l}\text { Family Income }>2 \text { Minimum Wages } \\
(N=142) \\
\text { Mean }( \pm \text { SD }) \mathrm{N}\end{array}$ & $\begin{array}{l}\text { Family Income }<2 \text { Minimum } \\
\text { Wages }(N=34) \\
\text { Mean }( \pm S D) N\end{array}$ & $\begin{array}{l}P \text { - } \\
\text { Value }\end{array}$ \\
\hline \multirow{2}{*}{$\begin{array}{l}\text { HD start before } 1500 \mathrm{Hrs} \\
(N=132)\end{array}$} & Steps & 3326 (2296) 108 & 3673 (2386) 24 & 0.255 \\
\hline & Sedentary Time & $546.3(152.1) 108$ & $504.1(120.4) 24$ & 0.029 \\
\hline \multirow[t]{2}{*}{$\begin{array}{l}\text { HD start after } 1500 \mathrm{Hrs} \\
(\mathrm{N}=48)\end{array}$} & Steps & 4620 (3127) 39 & 8491 (6891) 10 & $\begin{array}{l}< \\
0.0001\end{array}$ \\
\hline & Sedentary Time & 487.7 (119.9) 39 & $425.1(206.3) 10$ & 0.030 \\
\hline
\end{tabular}

PA levels on HD days by shift calculated based on each HD start time before or after $1500 \mathrm{~h}$. Among the 176 patients with accelerometry data, some patients stratified by age and income category received an HD treatment in both shifts during the 7 days of accelerometry

sedentary time by age categories in patients with HD starting after $1500 \mathrm{~h}$.

Another sub-group analysis of PA per $24 \mathrm{~h}$ on HD days by shift and family income level found no differences in step counts among patients starting HD before $1500 \mathrm{~h}$ with a family income above and below 2 minimum wages. However, patients starting HD before 1500 $\mathrm{h}$ with a family income $<2$ minimum wages had 42.2 fewer minutes of sedentary time per $24 \mathrm{~h}$ after HD as compared to patients with a family income $>2$ minimum wages $(p=0.029)$ (Table 6). Among patients with HD starting after $1500 \mathrm{~h}$, those with a family income $<2$ minimum wages took 3872 more steps $(p<0.0001)$ and had 62.6 fewer minutes of sedentary time $(p=0.030)$ per $24 \mathrm{~h}$ after HD versus patients with a family income $>2$ minimum wages.

Granular assessment of PA identified patients with HD starting before $1500 \mathrm{~h}$ performed 116, 1741, and 871 fewer steps during the 0-to-30-min, >11.0-to- $\leq$ 15.5 -h, and $>15.5$-to- $\leq 20.0$-h post-HD periods on HD days respectively versus patients with $\mathrm{HD}$ starting after $1500 \mathrm{~h}$ (all $p<0.05$ ) (Table 7). Despite this, patients with HD starting before $1500 \mathrm{~h}$ performed 68 and 680 more steps during the 91-to-120-min and > 2.0 -to- $\leq 6.5$-h post-HD periods versus patients with HD starting after $1500 \mathrm{~h}$ (both $p<0.05$ ). Similar patterns of PA were observed for MVPA. Minutes of sedentary time during the periods $2-\mathrm{h}$ post-HD did not differ between patients starting HD before versus after $1500 \mathrm{~h}$. Patients starting before $1500 \mathrm{~h}$ had more minutes of sedentary time in the $>2.0$-to- $\leq 11.0 \mathrm{~h}$ periods after dialysis HD (both $p<0.0001$ ) and less minutes of sedentary time in the $>11.0$-to- $\leq 20.0 \mathrm{~h}$ periods post-HD (both $p<0.05$ ) compared to those starting HD after $1500 \mathrm{~h}$.
PA levels on HD days were similar on the first (Monday/Tuesday), second (Wednesday/Thursday) or third (Friday/Saturday) HD session of the week and did not differ between groups in ANOVA and t-test comparisons (Table 8).

Granular steps did not differ by day of the week. Patients performed 0.4 more minutes of MVPA during the 91-to-120-min post-HD period on the first versus the third HD session of the week $(p=0.042)$; no other differences were observed (Table 9).

\section{Discussion}

This analysis of baseline data from the HDFIT trial characterized the profiles of PA in reference to the timing of $\mathrm{HD}$ and revealed unique PA patterns in the post-HD period, compared to concurrent times on subsequent non-HD days. Clinically stable prevalent HD patients in the first 2 years of HD who did not have limitations in ambulation tended to be highly sedentary, with levels of PA that only increased their metabolic energy expenditure rate by one tenth of a $\mathrm{kcal} / \mathrm{kg} /$ hour above the resting level. As anticipated, PA levels per $24 \mathrm{~h}$ were lower on HD days compared to the first and second non-HD days. PA levels and patterns per $24 \mathrm{~h}$ are consistent with other literature reports of PA measured on calendar days (i.e. 0:00-23:59 h) [7, 18-25]. Specialized accelerometry methods allowed us to characterize PA relative to the time of the start of HD. Lower PA levels on HD days were found to be primarily attributable to inactivity during HD, accounting for $>1000$ fewer steps on HD days. Contrary to our main hypothesis, patients had higher levels and the highest rates of PA within $2 \mathrm{~h}$ after HD as compared to concurrent times on subsequent non-HD days, yet this only accounted for about 300 additional steps. PA profiles differed in later HD shifts, with HD 
Table 7 Average Physical Activity (PA) Levels on HD Days Per Predefined Granular Slice Period by Dialysis Shift

\begin{tabular}{llll}
\hline Step Counts Per Predefined Granular Slice Period $(\boldsymbol{N}=176)$ & & \\
\hline Slice Period during/after HD & HD Start before 1500 Hrs $( \pm$ SD $)(\boldsymbol{N}=132)$ & HD Start after 1500 Hrs $( \pm S D)(\boldsymbol{N}=48)$ & $P$-Value vs before 1500 Hrs \\
\hline HD Period & $93(244)$ & $124(254)$ & 0.447 \\
$0.0-\leq 0.5 \mathrm{Hr}$ Post-HD & $229(231)$ & $345(347)$ & 0.011 \\
$>0.5-\leq 1.0 \mathrm{Hr}$ Post-HD & $288(231)$ & $247(191)$ & 0.270 \\
$>1.0-\leq 1.5 \mathrm{Hr}$ Post-HD & $254(194)$ & $202(210)$ & 0.119 \\
$>1.5-\leq 2.0 \mathrm{Hr}$ Post-HD & $194(179)$ & $126(176)$ & 0.025 \\
$>$ 2.0- $\leq 6.5 \mathrm{Hr}$ Post-HD & $949(798)$ & $269(319)$ & $<0.0001$ \\
$>6.5-\leq 11.0 \mathrm{Hr}$ Post-HD & $570(660)$ & $767(968)$ & 0.192 \\
$>11.0-\leq 15.5 \mathrm{Hr}$ Post-HD & $344(483)$ & $2085(2320)$ & $<0.0001$ \\
$>15.5-\leq 20.0 \mathrm{Hr}$ Post-HD & $968(1130)$ & $1839(1563)$ & $<0.0001$
\end{tabular}

MVPA Per Predefined Granular Slice Period $(N=176)$

\begin{tabular}{|c|c|}
\hline $\begin{array}{l}\text { Slice Period during/after } \\
\text { HD }\end{array}$ & $\begin{array}{l}\text { HD Start before } 1500 \text { Hrs }( \pm S D)(N= \\
132)\end{array}$ \\
\hline HD Period & $0.3(1.7)$ \\
\hline $0.0-\leq 0.5 \mathrm{Hr}$ Post-HD & $1.0(2.0)$ \\
\hline$>0.5-\leq 1.0 \mathrm{Hr}$ Post-HD & $1.2(2.0)$ \\
\hline$>1.0-\leq 1.5 \mathrm{Hr}$ Post-HD & $0.9(1.6)$ \\
\hline$>1.5-\leq 2.0 \mathrm{Hr}$ Post-HD & $0.7(1.4)$ \\
\hline$>2.0-\leq 6.5 \mathrm{Hr}$ Post-HD & $4.3(6.7)$ \\
\hline$>6.5-\leq 11.0 \mathrm{Hr}$ Post-HD & $2.7(4.0)$ \\
\hline$>11.0-\leq 15.5 \mathrm{Hr}$ Post-HD & $2.2(4.6)$ \\
\hline$>15.5-\leq 20.0 \mathrm{Hr}$ Post-HD & $5.1(8.6)$ \\
\hline
\end{tabular}

HD Start after $1500 \mathrm{Hrs}( \pm \mathrm{SD})(N=$ 48)

$0.5(1.7)$

$2.2(3.2)$

$1.1(1.7)$

$1.0(1.6)$

$0.6(1.3)$

$1.2(2.0)$

$4.5(6.2)$

$14.2(23.0)$

$11.0(16.2)$

HD Start after 1500 Hrs $( \pm$ SD) $(N=$ 132)

HD

HD Period $174.6(59.0)$

0.0- $\leq 0.5 \mathrm{Hr}$ Post-HD

$14.9(6.3)$

$>0.5-\leq 1.0 \mathrm{Hr}$ Post-HD

$11.2(6.2)$

$12.1(7.4)$

$14.6(6.6)$

$150.4(45.7)$

$106.1(62.1)$

$57.9(51.6)$

67.7 (49.0)

$>1.0-\leq 1.5 \mathrm{Hr}$ Post-HD

$>1.5-\leq 2.0 \mathrm{Hr}$ Post-HD

$>2.0-\leq 6.5 \mathrm{Hr}$ Post-HD

$>6.5-\leq 11.0 \mathrm{Hr}$ Post-HD

$>11.0-\leq 15.5 \mathrm{Hr}$ Post-HD

$>15.5-\leq 20.0 \mathrm{Hr}$ Post-HD

PA levels on $\mathrm{HD}$ days by shift calculated based an HD treatment in both shifts during the 7 days of accelerometry
48)

$192.6(52.3)$

$13.5(5.9)$

$11.1(5.6)$

$14.6(7.0)$

$16.0(7.4)$

$50.3(36.2)$

$34.9(50.0)$

$79.7(39.9)$

$114.4(45.5)$
$P$-Value vs before 1500

Hrs

0.605

0.005

0.747

0.773

0.4841

$<0.0001$

0.059

$<0.0001$

0.004

\section{$P$-Value vs before 1500}

Hrs

0.062

0.170

0.944

0.047

0.222

$<0.0001$

$<0.0001$

0.011

$<0.0001$

Table 8 Average Physical Activity (PA) Levels on HD Days Per 24 Hours by Dialysis Day of the Week

\begin{tabular}{lllll}
\hline HD Session of Week $(\boldsymbol{N}=176)$ & Steps Count Per 24 Hrs $( \pm \mathrm{SD})$ & $\boldsymbol{P}$-Value vs HD 1 & Minutes of MVPA Per 24 Hrs $( \pm \mathrm{SD})$ & $\boldsymbol{P}$-Value vs HD 1 \\
\hline HD 1 (Monday/Tuesday) & $3981(3096)$ & Reference & $21.0(27.9)$ & Reference \\
HD 2 (Wednesday/Thursday) & $3976(3289)$ & 0.987 & $20.5(30.5)$ & 0.872 \\
HD 3 (Friday/Saturday) & $3820(3270)$ & 0.637 & $19.1(32.5)$ & 0.560 \\
\hline
\end{tabular}


Table 9 Average Physical Activity (PA) Levels on HD Days Per Predefined Granular Slice Period by Dialysis Day of the Week

\begin{tabular}{|c|c|c|c|c|c|}
\hline \multicolumn{6}{|c|}{ Step Counts Per Predefined Granular Slice Period $(\boldsymbol{N}=176)$} \\
\hline Slice Period during/after HD & $\mathrm{HD} 1( \pm \mathrm{SD})$ & $\mathrm{HD} 2( \pm \mathrm{SD})$ & $\boldsymbol{P}$-Value vs HD 1 & $\mathrm{HD} 3( \pm \mathrm{SD})$ & $\boldsymbol{P}$-Value vs HD 1 \\
\hline HD Period & $70(142)$ & $133(550)$ & 0.155 & $83(183)$ & 0.460 \\
\hline $0.0-\leq 0.5 \mathrm{Hr}$ Post-HD & $255(300)$ & $273(311)$ & 0.586 & $265(337)$ & 0.766 \\
\hline$>0.5-\leq 1.0 \mathrm{Hr}$ Post $-\mathrm{HD}$ & $272(294)$ & $287(277)$ & 0.633 & $293(336)$ & 0.945 \\
\hline$>1.0-\leq 1.5 \mathrm{Hr}$ Post-HD & $243(246)$ & $252(252)$ & 0.742 & $247(256)$ & 0.885 \\
\hline$>1.5-\leq 2.0 \mathrm{Hr}$ Post-HD & $205(247)$ & $191(278)$ & 0.629 & $170(203)$ & 0.170 \\
\hline$>2.0-\leq 6.5 \mathrm{Hr}$ Post-HD & $808(814)$ & $816(1002)$ & 0.936 & $858(1137)$ & 0.655 \\
\hline$>6.5-\leq 11.0 \mathrm{Hr}$ Post-HD & $568(758)$ & $675(1087)$ & 0.363 & $645(774)$ & 0.426 \\
\hline$>11.0-\leq 15.5$ Hr Post-HD & $1132(2038)$ & $1081(2078)$ & 0.851 & $1047(1971)$ & 0.752 \\
\hline$>15.5-\leq 20.0 \mathrm{Hr}$ Post-HD & $1441(1588)$ & $1337(1585)$ & 0.598 & $1253(1649)$ & 0.355 \\
\hline \multicolumn{6}{|c|}{ MVPA Per Predefined Granular Slice Period $(N=176)$} \\
\hline Slice Period during/after HD & HD 1 ( $\pm S D)$ & HD 2 ( $( \pm \mathrm{SD})$ & $P$-Value vs HD 1 & HD 3 ( \pm SD) & $P$-Value vs HD 1 \\
\hline HD Period & $0.4(1.2)$ & $0.5(4.3)$ & 0.656 & $0.2(0.9)$ & 0.059 \\
\hline $0.0-\leq 0.5 \mathrm{Hr}$ Post-HD & $1.4(2.8)$ & $1.3(2.9)$ & 0.665 & $1.3(2.9)$ & 0.642 \\
\hline$>0.5-\leq 1.0 \mathrm{Hr}$ Post-HD & $1.2(2.5)$ & $1.2(2.5)$ & 0.551 & $1.1(3.1)$ & 0.762 \\
\hline$>1.0-\leq 1.5 \mathrm{Hr}$ Post-HD & $1.0(2.3)$ & $0.9(1.8)$ & 0.570 & $0.9(2.0)$ & 0.520 \\
\hline$>1.5-\leq 2.0 \mathrm{Hr}$ Post-HD & $0.9(2.2)$ & $0.8(2.3)$ & 0.840 & $0.5(1.3)$ & 0.042 \\
\hline$>2.0-\leq 6.5 \mathrm{Hr}$ Post-HD & $3.5(6.4)$ & $3.4(7.9)$ & 0.970 & $4.4(11.2)$ & 0.336 \\
\hline$>6.5-\leq 11.0 \mathrm{Hr}$ Post-HD & $2.9(4.9)$ & $2.9(6.3)$ & 0.944 & $3.4(6.5)$ & 0.501 \\
\hline$>11.0-\leq 15.5$ Hr Post-HD & $7.5(19.1)$ & $7.5(18.5)$ & 0.998 & $7.2(19.8)$ & 0.905 \\
\hline$>15.5-\leq 20.0 \mathrm{Hr}$ Post-HD & $8.1(14.1)$ & $8.2(16.6)$ & 0.947 & $6.2(14.4)$ & 0.275 \\
\hline
\end{tabular}

staring after $1500 \mathrm{~h}$ being associated with patients taking $>2000$ more steps $24 \mathrm{~h}$ after HD. These distinct levels were driven by activity performed within $30 \mathrm{~min}$ after $\mathrm{HD}$ and 11-to-20 $\mathrm{h}$ after HD. Although the findings might be representative of late shift patients being a more functional subgroup who perform more daily activities, age and family income level did not appear to be influencing the distinctions in PA levels between earlier and later HD shifts. The distinctions in PA performed 11-to-20 $\mathrm{h}$ after HD may also be attributed to the timing of sleep after HD in the later shift patients and PA patterns performed the subsequent day.

The literature reports relatively sedentary levels of PA in the HD population ( $<5000 \mathrm{steps} /$ day, $<21.5 \mathrm{~min}$ of MVPA/day, <3.0 MET (kcal/kg/hour) or equivalent measures), however, large variations exist (differences up to $6000+$ steps/day) depending on populations and geographies [1, 2, 6, 7, 18-25, 39]. Our findings are consistent with HD patients generally performing < 4000 steps per 24-h period on HD days, and about 5000 steps per 24-h period on non-HD days. Despite this, patients achieved goals for MVPA on non-HD days in line with WHO recommendations of $\geq 150 \mathrm{~min}$ of $\mathrm{MVPA} /$ week [39]. However, MET levels were only $0.1 \mathrm{kcal} / \mathrm{kg} / \mathrm{hour}$ above the resting level.
Contrary to our expectation, PA was higher following HD. This is an unexpected finding given patientreported dialysis recovery time is perceived to be longer than $2 \mathrm{~h}$ for more than $65 \%$ of patients and recovery time associates negatively with physical component summary scores of the Kidney Disease Quality of Life (KDQOL) survey [40]. However, consistent with our findings, a small accelerometry study using the RT-3 monitor (StayHealthy, Monrovia, CA) among $20 \mathrm{HD}$ patients has previously found raw vector magnitude acceleration counts per minute (CPM) were significantly higher within the $2 \mathrm{~h}$ period following dialysis on HD days, as compared to the same time on non-HD days [41]. Despite that the accelerometer used was unable to compute step counts for generalizability, nor filter raw acceleration data, this study's results and ours showed consistent signals of a potentially important timepoint for more PA being performed proximal to dialysis. Future investigations appear warranted to investigate potential inverse associations in patient perceptions of self-reported dialysis recovery time and measured PA post-HD. Also, given this RCT included a select population, further investigations are needed to confirm our findings are generalizable in the overall HD population. 
Increased post-HD PA might be associated with transportation from the clinic, especially in the first hour when step counts and rates are the highest. Post-HD PA levels appear to be influenced by transportation type with patients using public transportation and walking performing about 600 more steps during the 2-h period after HD compared to those using of a family car, taxi, and ambulance; this finding coincides with no differences in sedentary time between groups up to $90 \mathrm{~min}$ after $\mathrm{HD}$, and only 3 min less sedentary time in those using public transportation and walking in the prespecified period 90-to-120 min after HD. Other contributing factors may include improved fluid and electrolyte status after HD, and routine activities performed after HD (i.e. cooking, shopping, errands), which may associate to increased levels of light PA observed 31-to-90 min after HD. The finding that PA rates are the highest in the first hours after HD might be signaling an important timepoint in the post-HD period that warrants investigation in the future. It has been recognized sudden death typically occurs within $12 \mathrm{~h}$ of HD [42]. However, it is unknown if higher PA rates could cause hemodynamic stress immediately following HD, which itself inherently causes a hemodynamic challenge [43].

HD schedules before/after $1500 \mathrm{~h}$ might also influence PA on HD days. Compared to patients starting HD before $1500 \mathrm{~h}$, those treated after $1500 \mathrm{~h}$ presented higher step counts at $30 \mathrm{~min}$ and 11-to-20 h after HD. Despite this, patients starting HD before $1500 \mathrm{~h}$ took more steps 1.5-to-6.5 h after HD compared to patients starting HD after $1500 \mathrm{~h}$; relatively consistent signals were observed with MVPA and sedentary time. We found younger patients ( $<65$ years old) treated in earlier HD shifts performed $>1600$ more steps per $24 \mathrm{~h}$ than patients $\geq 65$ years old with no differences by age in sedentary time. However, step counts and sedentary time did not significantly differ by age in later HD shift patients. Although the income level was not associated with distinctions in step counts for earlier HD shift patients, those with a low family income that were treated in earlier shifts had about 40 fewer minutes of sedentary time per $24 \mathrm{~h}$ after HD. Among later shift patients, a low family income level was associated with patients taking about 3800 more steps and having about 60 less minutes of sedentary time per $24 \mathrm{~h}$. Despite this, within sub-group differences for age and family income sub-groups were relatively consistent with changes in the overall cohort. Our findings could possibly be related to free living PA and sleep patterns, as well as other patient characteristics; a selection bias may be contributing to the findings. Patients treated on the first HD shift have been previously found to be associated to altered sleep patterns, [6, 7] and the patterns and timing of sleep between patients treated in earlier and later shifts may be reflected in the distinctions in PA found $90 \mathrm{~min}$ to $20 \mathrm{~h}$ after HD. The HD day of the week did not remarkably affect PA.

PA is of utmost importance to improving/maintaining health in all populations, and it is a useful surrogate marker of negative outcomes. The United States guidelines for PA suggest performing any higher quantity of PA yields some health benefits [44]. Select European and Oceania countries have adopted the concept that exercise is "medicine" for dialysis patients and should be prescribed, but unfortunately, this philosophy has not been adopted in many countries in the world [45]. General and elderly populations have been shown to exhibit an inverse relationship between steps taken and outcomes [46-50]. Achievement of increases of 1000 more steps per day associates with about a $5 \%$ lower cardiovascular event rate and a $15 \%$ lower all-cause mortality rate [46, 47]. A 7-year observational study in HD patients found taking $\geq 5000$ steps a day associates with an 18.5 percentage point improvement in the cumulative survival rate compared to having < 5000 steps daily [24]. In addition, this study found patients who had a $30 \%$ decrease in daily steps had a 3-fold higher adjusted risk of death compared to patients with a $30 \%$ increase [24].

Intradialytic exercise programs could potentially increase PA profiles on HD days. We established patients perform the highest levels and rates of activity within $2 \mathrm{~h}$ of $\mathrm{HD}$, the requirements of sitting/lying during the HD treatment was found to be the primary cause of higher inactivity on HD days, which is consistent with, confirms, and builds upon findings from a small prior accelerometry study [41]. Clinicians, care teams and the nephrology community should recognize HD regimens are a major factor negatively impacting activity levels. A RCT has shown 4 months of intradialytic pedaling exercise decreased arterial stiffness [51]. Although intradialytic exercise such as cycling/pedaling may be a low hanging fruit for targeting interventions, with a higher potential to yield beneficial health outcomes, it can only reach patients $12 \mathrm{~h}$ per week. Developing both intradialytic and interdialytic exercise programs should be a high priority for providers and clinicians as a means to increase PA in this largely sedentary population.

We believe it will be of importance for clinicians to prescribe exercise as a medicine in a patient centric manner and routinely track adherence. Programs can be simple and not require additional resources, such as prescribing a 20 min walk every day and leg lifts/stretching/ Pilates during each HD treatment. It may be important to consider small incremental increases that focus on HD patients performing more activity, rather than achieving a specific exercise intensity. Our findings among patients without any impaired mobility/ambulation can be applied to the design of interventions aiming to increase PA in similar HD populations. They suggest 
the timing of interdialytic exercise on HD days might be most optimal to be recommended before or $>2 \mathrm{~h}$ after HD when PA levels tend to be lower. It appears relevant to tailor exercise recommendations based on transportation type, with an emphasis of efforts in patients using a car to come to/from the clinic. On days without HD, exercise could be encouraged at the patient's discretion. Patients treated in earlier HD shifts are possibly an important sub-group of patients to target with exercise interventions. Among this sub-group, older patients performed the lowest PA, yet younger patients were also highly inactive taking $<4000$ steps per $24 \mathrm{~h}$. Therefore, age may not be an appropriate consideration in targeting exercise interventions if no other contraindications to performing PA exist.

While our study has many strengths including use of high-quality RCT data, there are some limitations. Patients may not be representative of the overall HD population, but specifically generalizable to patients in the first 2 years of HD without impaired mobility/ambulation who are adherent with HD. We used self-reported HD times which may be inaccurate. There is also a potential for undercounting of PA using ActiLife software default algorithm, which has been validated in younger people performing exercise [37, 38]. Nonetheless, accelerometry is internally consistent and PA levels were found to be similar with previous reports [7, 18-25]. Rates of PA were calculated by period without adjustment for sleep time, which was excluded per protocol. Although this does not influence absolute values of PA, it could affect normalized rates.

\section{Conclusions}

In conclusion, the findings identify patients performed higher PA levels and the highest PA rates in the 2-h postHD period on HD days compared to concurrent times on non-HD days, however, the requirement of sitting/lying during HD causes overall 24-h PA levels on HD days to be lower than non-HD days. Timing of the HD session associates to distinct PA levels, but further investigations are needed. Exercise programs should be developed that increase activity during and between HD treatments.

\section{Supplementary information}

Supplementary information accompanies this paper at https://doi.org/10 1186/s12882-020-01853-2.

Additional file 1: Appendix A. HDFIT Study Site Investigators and Trial Leadership; Appendix B. EPICENTER ACRO HDFIT Key Leadership and Affiliates; Appendix C. HDFIT Steering Committee.

\section{Abbreviations}

ACRO: Academic clinical research organization; ANOVA: Analysis of variance BUN: Blood urea nitrogen; CKD: Chronic kidney disease; CPM: Counts per minute; DBP: Diastolic blood pressure; EPICENTER: Center for Epidemiology and Clinical Research; ESKD: End stage kidney disease; HD: Hemodialysis;
HDF: Hemodiafiltration; iPTH: Intact parathyroid hormone; Kt/N: Dialysis adequacy; MET: Metabolic rate; MVPA: Moderate to vigorous physical activity; PA: Physical activity; PUCPR: Pontifícia Universidade Católica do Paraná; RCT: Randomized controlled trial; SBP: Systolic blood pressure

\section{Acknowledgements}

We would like to acknowledge and thank the site investigators, participating dialysis centers and staff conducting this trial listed in Additional File 1 Appendix A; the EPICENTER ACRO staff and affiliates managing the trial listed in Additional File 1 Appendix B; and the external advisory committee members Bernard Canaud, MD, PhD, Cristina Marelli, MD, and Rodrigo S. Reis, $\mathrm{PhD}, \mathrm{MSC}$

For the HDFIT Study Investigators: Refer to the Additional File 1. Consortia: For the HDFIT Study Investigators.

Ana Claudia Dambiski, Thaylane Amanda de Souza, Daniela Ponce, Edwa Maria Bucuvic, Luciana Menin Ferreira, Wanderson de Souza Carvalho, Jorge Paulo Strogoff de Matos, Esther Oliveria Silva, Manuel Carlos Martins de Castro, Celina de Fátima e Silva, Maria Eugenia F Canziani, Silvia R Manfredi, Katia Santos, Ana Paula Fonseca Correia, Giovani Gadonski, Adriana Conti, Inah Pecly, Camille Souza Paixão, Viviane Calice-Silva, Simone Ribeiro, Lizia Regina Ribeiro Caldeira, Adailto Santos, Rosilene Motta Elias, Andreia Barbosa Dos Santos, Américo Lourenço Cuvello-Neto, Amanda Monteiro Virolli.

\section{Authors' contributions}

Trial procedures were performed under the oversight of the principal investigator (RPF) and site investigators listed in Additional File 1 Appendix A. The design and conduct of the study was performed by: RPF, CEPF, ALCN, ABLB, TPM, PBG, MEFC and the HDFIT investigators and research staff listed in Additional File 1 Appendix A and EPICENTER ACRO and affiliated staff listed in Additional File 1 Appendix B. The data collection, analytical design, and analysis for this study was conducted by: RPF, TPM, JWL, PBG, MG, HH, $\mathrm{MH}, \mathrm{LAU}, \mathrm{PK}$, JGR. The intellectual content, interpretation, drafting and revision of this manuscript was performed by all authors, the HDFIT investigators listed in Additional File 1 Appendix A and steering committee listed in Additional File 1 Appendix C. The decision to submit this manuscript for publication was jointly made by all authors and parties. The manuscript was confirmed to be accurate and approved by all authors.

\section{Funding}

This trial was a multi-center investigator-initiated study, whereby the site investigators and principal investigator were not being monetary funded for the conduct of study activities. This project was supported by: 1) the study investigators, 2) the proponent institution Pontifícia Universidade Católica do Paraná, 3) the outpatient dialysis centers, and 4) Fresenius Medical Care. The steering committee was comprised of nephrologists representing site institutions and supporting affiliates.

Investigators were involved in the design of the protocol and performed medical oversight and the coordination of data collection during the trial. The principal investigator provided medical oversight of the conduct of the trial at all sites under the guidance of the steering committee and coordinated the trial management.

The proponent institution Pontifícia Universidade Católica do Paraná supported the trial with infrastructure for study management through use of the university's ACRO, hosting of the REDCap electronic case report form on the university's server, and use of the university's central Ethics Review Board and Research Council.

The outpatient dialysis centers permitted clinical research at the clinics and supported the trial with their clinical staff who performed data collection and the conduct of study procedures under the oversight of the site investigators and local trial leadership.

Fresenius Medical Care provided the sites with the infrastructure for the conduct of the trial including HDF machines, dialysis supplies for study participants, body composition monitor machines in clinics without them. Also, they provided some staff for site monitoring. Fresenius Medical Care provided a monetary award to PUCPR'S ACRO (EPICENTER) that performed the central management, data acquisition, and monitoring. Fresenius Medical Care and the subsidiary company Renal Research Institute provided support from statistical experts to assist in the analysis of trial data under the oversight of the steering committee. Fresenius Medical Care has supported three investigator meetings, as well as three steering committee meetings. 
The leadership of Fresenius Medical Care reviewed and approved the protocol prior to commencement.

The steering committee members who represent supporting institutions reviewed and approved the research design, protocol, addendums and changes to the protocol, analyses, and this publication of study data, as well as provided oversight of the trial conduct and safety.

\section{Availability of data and materials}

The original source documents and electronic datasets, from the trial, as well as the coding from this analysis are not publicly available. The original trial documents, datasets, and coding are regulated and maintained by the EPICENTER ACRO. Affiliated investigators can access the trial dataset under oversight and approval of the HDFIT steering committee.

\section{Ethics approval and consent to participate}

The HDFIT trial is was performed under a protocol approved by Pontifícia Universidade Católica do Paraná Ethics Review Board (Central tracking \#: 54926916.7.1001.0020; Approval \#: 1.538.784). All participants had provided written informed consent prior to participation in any study activities.

\section{Consent for publication}

Not applicable.

\section{Competing interests}

JWL, MH, MG are students at Pontifícia Universidade Católica do Paraná. JWL, $\mathrm{HH}, \mathrm{LAU}$, are employees of Fresenius Medical Care. MH, PK, JGR are employees of Renal Research Institute, a wholly owned subsidiary of Fresenius Medical Care North America. PBG receives travel support from Fresenius Medical Care. CEPF, ALCN receive consulting fees and speaker honorarium from Fresenius Medical Care. CEPF receives lecture fees and travel support from Fresenius Medical Care, Alexion, Baxter, and Astra Zeneca, and is employed by Pontifícia Universidade Católica do Rio Grande do Sul. ABLB is an employee of Fresenius Medical Care Brazil. LAU, PK have share options/ownership in Fresenius Medical Care. PK receives author honorarium from Up-To-Date and is on the Editorial Board of Blood Purification and Kidney and Blood Pressure Research. RPF, TPM are employed by Pontifícia Universidade Católica do Paraná. RPF, CEPF, TPM, MEFC are recipients of scholarships from the Brazilian Council for Research (CNPq). MEFC is employee by Federal University of São Paulo, and receives research grants, consulting fees, and honoraria from Baxter Healthcare and Fresenius Medical Care. RPF is employed by Arbor Research Collaborative for Health, and receives research grants, consulting fees, and honoraria from Astra Zeneca, Novo Nordisc, Akebia, and Fresenius Medical Care. All other authors declare no relevant conflicts of interest.

\section{Author details}

'School of Medicine, Pontifícia Universidade Católica do Paraná, Imaculada Conceição, 1155, Curitiba, PR 80215-901, Brazil. 'Global Medical Office, Fresenius Medical Care, Waltham, MA, USA. ${ }^{3}$ Research Division, Renal Research Institute, New York, NY, USA. ${ }^{4}$ Health Technology Graduate Program, Pontifícia Universidade Católica do Paraná, Curitiba, PR, Brazil. ${ }^{5}$ School of Medicine, Pontifícia Universidade Católica do Rio Grande do Sul, Porto Alegre, RS, Brazil. 'Hospital Alemão Oswaldo Cruz, São Paulo, SP, Brazil. ${ }^{7}$ Fresenius Medical Care Brazil, São Paulo, SP, Brazil. ${ }^{8}$ Icahn School of Medicine at Mount Sinai, New York, NY, USA. ' Universidade Federal de São Paulo, São Paulo, SP, Brazil.

Received: 1 October 2019 Accepted: 12 May 2020

Published online: 25 May 2020

\section{References}

1. Physical Activity Guidelines Advisory Committee report, 2008. To the secretary of health and human services. Part a: executive summary. Nutr Rev. 2009;67(2):114-20.

2. Tudor-Locke C, Craig CL, Aoyagi Y, Bell RC, Croteau KA, De Bourdeaudhuij I, Ewald B, Gardner AW, Hatano Y, Lutes LD, et al. How many steps/day are enough? For older adults and special populations. Int J Behav Nutr Phys Act. 2011;8:80

3. Lopes AA, Lantz B, Morgenstern H, Wang M, Bieber BA, Gillespie BW, Li Y, Painter $\mathrm{P}$, Jacobson SH, Rayner HC, et al. Associations of self-reported physical activity types and levels with quality of life, depression symptoms, and mortality in hemodialysis patients: the DOPPS. Clin J Am Soc Nephrol. 2014;9(10):1702-12.

4. Morishita S, Tsubaki A, Shirai N. Physical function was related to mortality in patients with chronic kidney disease and dialysis. Hemodial Int. 2017;21(4): 483-89.

5. Tudor-Locke C, Washington TL, Hart TL. Expected values for steps/day in special populations. Prev Med. 2009;49(1):3-11.

6. Han M, Williams S, Mendoza M, Ye X, Zhang H, Calice-Silva V, Thijssen S, Kotanko P, Meyring-Wosten A. Quantifying physical activity levels and sleep in hemodialysis patients using a commercially available activity tracker. Blood Purif. 2016;41(1-3):194-204.

7. Williams S, Han M, Ye X, Zhang H, Meyring-Wosten A, Bonner M, Young C, Thijssen S, Marsh D, Kotanko P. Physical activity and sleep patterns in hemodialysis patients in a suburban environment. Blood Purif. 2017;43(1-3): 235-43.

8. Rosa CS, Bueno DR, Souza GD, Gobbo LA, Freitas IF Jr, Sakkas GK, Monteiro HL. Factors associated with leisure-time physical activity among patients undergoing hemodialysis. BMC Nephrol. 2015;16:192.

9. Johansen KL, Chertow GM, Ng AV, Mulligan K, Carey S, Schoenfeld PY, KentBraun JA. Physical activity levels in patients on hemodialysis and healthy sedentary controls. Kidney Int. 2000;57(6):2564-70.

10. Afsar $B$. The relationship between intact parathyroid hormone levels and daily physical activity in hemodialysis patients. Int J Artif Organs. 2014;37(5):350-7.

11. Zamojska S, Szklarek M, Niewodniczy M, Nowicki M. Correlates of habitual physical activity in chronic haemodialysis patients. Nephrol Dial Transplant. 2006;21(5):1323-7.

12. van Sandwijk MS, Al Arashi D, van de Hare FM, van der Torren JMR, Kersten MJ, Bijlsma JA, IJM TB, Bemelman FJ. Fatigue, anxiety, depression and quality of life in kidney transplant recipients, haemodialysis patients, patients with a haematological malignancy and healthy controls. Nephrol Dial Transplant. 2019;34(5):833-838.

13. Alavi NM, Aliakbarzadeh Z, Sharifi K. Depression, anxiety, activities of daily living, and quality of life scores in patients undergoing renal replacement therapies. Transplant Proc. 2009;41(9):3693-6.

14. Kutner NG, Zhang R, Allman RM, Bowling CB. Correlates of ADL difficulty in a large hemodialysis cohort. Hemodial Int. 2014;18(1):70-7.

15. Han M, Ye X, Preciado P, Williams S, Campos I, Bonner M, Young C, Marsh D, Larkin JW, Usvyat LA, et al. Relationships between neighborhood walkability and objectively measured physical activity levels in hemodialysis patients. Blood Purif. 2018;45(1-3):236-44.

16. Flythe JE, Hilliard T, Castillo G, Ikeler K, Orazi J, Abdel-Rahman E, Pai AB, Rivara MB, St Peter WL, Weisbord SD, et al. Symptom prioritization among adults receiving in-center hemodialysis: a mixed methods study. Clin J Am Soc Nephrol. 2018;13(5):735-45.

17. Bossola M, Di Stasio E, Marzetti E, De Lorenzis K, Pepe G, Vulpio C. Fatigue is associated with high prevalence and severity of physical and emotional symptoms in patients on chronic hemodialysis. Int Urol Nephrol. 2018;50(7): 1341-6.

18. Katayama A, Miyatake N, Nishi H, Ujike K, Hashimoto H, Kurato R, Koumoto K. Relationship between changes in physical activity and changes in healthrelated quality of life in patients on chronic hemodialysis with 1-year followup. Acta Med Okayama. 2016;70(5):353-61.

19. Gomes EP, Reboredo MM, Carvalho EV, Teixeira DR, Carvalho LF, Filho GF, de Oliveira JC, Sanders-Pinheiro H, Chebli JM, de Paula RB, et al. Physical activity in hemodialysis patients measured by Triaxial accelerometer. Biomed Res Int. 2015;2015:645645.

20. Carvalho EV, Reboredo MM, Gomes EP, Teixeira DR, Roberti NC, Mendes JO, Oliveira JC, Sanders-Pinheiro H, Pinheiro BV. Physical activity in daily life assessed by an accelerometer in kidney transplant recipients and hemodialysis patients. Transplant Proc. 2014;46(6):1713-7.

21. Shibata S, Tsutou A, Shiotani H. Relation between sleep quality and daily physical activity in hemodialysis outpatients. Kobe J Med Sci. 2014;59(5):E161-6.

22. Matsuzawa R, Matsunaga A, Kutsuna T, Ishii A, Abe Y, Yoneki K, Harada M, Ishibashi M, Takeuchi Y, Yoshida A, et al. Association of habitual physical activity measured by an accelerometer with high-density lipoprotein cholesterol levels in maintenance hemodialysis patients. ScientificWorldJournal. 2013;2013:780783.

23. Avesani CM, Trolonge S, Deleaval P, Baria F, Mafra D, Faxen-Irving G, Chauveau P, Teta D, Kamimura MA, Cuppari L, et al. Physical activity and energy expenditure in haemodialysis patients: an international survey. Nephrol Dial Transplant. 2012;27(6):2430-4. 
24. Shimoda T, Matsuzawa R, Yoneki K, Harada M, Watanabe T, Matsumoto M, Yoshida A, Takeuchi Y, Matsunaga A. Changes in physical activity and risk of all-cause mortality in patients on maintence hemodialysis: a retrospective cohort study. BMC Nephrol. 2017;18(1):154.

25. Cupisti A, Capitanini A, Betti G, D'Alessandro C, Barsotti G. Assessment of habitual physical activity and energy expenditure in dialysis patients and relationships to nutritional parameters. Clin Nephrol. 2011;75(3):218-25.

26. Oishi D, Koitabashi K, Hiraki K, Imai N, Sakurada T, Konno Y, Shibagaki Y, Yasuda T, Kimura K. Physical activity is associated with serum albumin in peritoneal dialysis patients. Adv Perit Dial. 2012;28:148-52.

27. Masuda R, Imamura H, Mizuuchi K, Miyahara K, Kumagai $H$, Hirakata $H$. Physical activity, high-density lipoprotein cholesterol subfractions and lecithin:cholesterol acyltransferase in dialysis patients. Nephron Clin Pract. 2009;111(4):c253-9.

28. Pecoits-Filho R. Impact of HemoDiaFlltration on physical activity and selfreported outcomes (HDFIT). ClinicalTrialsgov Registry. 2016; https:// clinicaltrials.gov/ct2/show/NCT02787161 (Accessed 29 Sep 2019).

29. Pecoits-Filho R, Larkin JW, Poli-de-Figueiredo CE, Cuvello Neto AL, Barra AB, Canhada S, de Campos LG, Woehl J, Gonçalves PB, Han H, et al. Design and methodology of the impact of HemoDiaFIITration on physical activity and self-reported outcomes: a randomized controlled trial (HDFIT trial) in Brazil. BMC Nephrol. 2019;20(1):98.

30. Plasqui G, Westerterp KR. Physical activity assessment with accelerometers: an evaluation against doubly labeled water. Obesity (Silver Spring). 2007; 15(10):2371-9.

31. Yang CC, Hsu YL. A review of accelerometry-based wearable motion detectors for physical activity monitoring. Sensors (Basel). 2010;10(8):7772-88.

32. Evenson KR, Buchner DM, Morland KB. Objective measurement of physical activity and sedentary behavior among US adults aged 60 years or older. Prev Chronic Dis. 2012;9:E26.

33. O'Neill B, McDonough SM, Wilson JJ, Bradbury I, Hayes K, Kirk A, Kent L, Cosgrove D, Bradley JM, Tully MA. Comparing accelerometer, pedometer and a questionnaire for measuring physical activity in bronchiectasis: a validity and feasibility study? Respir Res. 2017;18(1):16.

34. Daugirdas JT. Second generation logarithmic estimates of single-pool variable volume Kt/N: an analysis of error. J Am Soc Nephrol. 1993;4(5):1205-13.

35. Choi L, Liu Z, Matthews CE, Buchowski MS. Validation of accelerometer wear and nonwear time classification algorithm. Med Sci Sports Exerc. 2011;43(2): 357-64.

36. Migueles JH, Cadenas-Sanchez C, Ekelund U, Delisle Nystrom C, MoraGonzalez J, Lof M, Labayen I, Ruiz JR, Ortega FB. Accelerometer data collection and processing criteria to assess physical activity and other outcomes: a systematic review and practical considerations. Sports Med. 2017;47(9):1821-45.

37. Sasaki JE, John D, Freedson PS. Validation and comparison of ActiGraph activity monitors. J Sci Med Sport. 2011;14(5):411-6.

38. Freedson PS, Melanson E, Sirard J. Calibration of the computer science and applications, Inc accelerometer. Med Sci Sports Exerc. 1998;30(5):777-81.

39. Schubert N, Evans R, Battye K, Gupta TS, Larkins S, Mclver L. International approaches to rural generalist medicine: a scoping review. Hum Resour Health. 2018;16(1):62

40. Rayner HC, Zepel L, Fuller DS, Morgenstern H, Karaboyas A, Culleton BF, Mapes DL, Lopes AA, Gillespie BW, Hasegawa T, et al. Recovery time, quality of life, and mortality in hemodialysis patients: the Dialysis outcomes and practice patterns study (DOPPS). Am J Kidney Dis. 2014;64(1):86-94.

41. Majchrzak KM, Pupim LB, Chen K, Martin CJ, Gaffney S, Greene JH, Ikizler TA. Physical activity patterns in chronic hemodialysis patients: comparison of dialysis and nondialysis days. J Ren Nutr. 2005;15(2):217-24.

42. Bleyer AJ, Hartman J, Brannon PC, Reeves-Daniel A, Satko SG, Russell G. Characteristics of sudden death in hemodialysis patients. Kidney Int. 2006; 69(12):2268-73.

43. Lertdumrongluk P, Streja E, Rhee CM, Sim JJ, Gillen D, Kovesdy CP, KalantarZadeh K. Changes in pulse pressure during hemodialysis treatment and survival in maintenance dialysis patients. Clin J Am Soc Nephrol. 2015;10(7):1179-91.

44. Piercy KL, Troiano RP. Physical activity guidelines for Americans from the US Department of Health and Human Services. Circ Cardiovasc Qual Outcomes. 2018;11(11):e005263.

45. Parker K. Intradialytic exercise is medicine for hemodialysis patients. Curr Sports Med Rep. 2016;15(4):269-75.

46. Kraus WE, Janz KF, Powell KE, Campbell WW, Jakicic JM, Troiano RP, Sprow K, Torres A, Piercy KL. Physical activity guidelines advisory C: daily step counts for measuring physical activity exposure and its relation to health. Med Sci Sports Exerc. 2019;51(6):1206-12.

47. Lee IM, Shiroma EJ, Kamada M, Bassett DR, Matthews CE, Buring JE. Association of Step Volume and Intensity With All-Cause Mortality in Older Women. JAMA Intern Med. 2019;179(8):1105-1112.

48. Dwyer T, Pezic A, Sun C, Cochrane J, Venn A, Srikanth V, Jones G, Shook RP, Sui $X$, Ortaglia A, et al. Objectively measured daily steps and subsequent long term all-cause mortality: the Tasped prospective cohort study. PLoS One. 2015;10(11):e0141274.

49. Yamamoto N, Miyazaki H, Shimada M, Nakagawa N, Sawada SS, Nishimuta M, Kimura Y, Kawakami R, Nagayama H, Asai H, et al. Daily step count and all-cause mortality in a sample of Japanese elderly people: a cohort study. BMC Public Health. 2018;18(1):540.

50. Jefferis BJ, Parsons TJ, Sartini C, Ash S, Lennon LT, Papacosta O, Morris RW Wannamethee SG, Lee IM, Whincup PH. Objectively measured physical activity, sedentary behaviour and all-cause mortality in older men: does volume of activity matter more than pattern of accumulation? $\mathrm{Br} J$ Sports Med. 2019:53(16):1013-1020.

51. Cooke AB, Ta V, lqbal S, Gomez YH, Mavrakanas T, Barre P, Vasilevsky M, Rahme E, Daskalopoulou SS. The impact of Intradialytic pedaling exercise on arterial stiffness: a pilot randomized controlled trial in a hemodialysis population. Am J Hypertens. 2018;31(4):458-66.

\section{Publisher's Note}

Springer Nature remains neutral with regard to jurisdictional claims in published maps and institutional affiliations.

\section{Ready to submit your research? Choose BMC and benefit from:}

- fast, convenient online submission

- thorough peer review by experienced researchers in your field

- rapid publication on acceptance

- support for research data, including large and complex data types

- gold Open Access which fosters wider collaboration and increased citations

- maximum visibility for your research: over $100 \mathrm{M}$ website views per year

At BMC, research is always in progress.

Learn more biomedcentral.com/submissions 